PHYSICAL REVIEW D 83, 114512 (2011)

\title{
Brillouin improvement for Wilson fermions
}

\author{
Stephan Dürr ${ }^{1,2}$ and Giannis Koutsou ${ }^{1,2}$ \\ ${ }^{1}$ Bergische Universität Wuppertal, Gaußstraße 20, 42119 Wuppertal, Germany \\ ${ }^{2}$ Jülich Supercomputing Center, Forschungszentrum Jülich, 52425 Jülich, Germany
}

(Received 11 January 2011; published 21 June 2011)

\begin{abstract}
We present a parameter-free Wilson-type lattice Dirac operator with an 81-point stencil for the covariant derivative and the Laplacian, which attempts to minimize the breaking of rotational symmetry near the boundary of the Brillouin zone. The usefulness of this "Brillouin operator" in practical applications is explored by studying the scaling of pseudoscalar decay constants in quenched QCD, with rather good results in the physical charm region. We also investigate the suitability of this operator as a kernel to the overlap procedure. Here, the resulting overlap operator is found to be cheaper to construct and significantly better localized than the variety with the standard Wilson kernel.
\end{abstract}

DOI: 10.1103/PhysRevD.83.114512

PACS numbers: 11.15.Ha, 12.38.Gc, 13.20.Fc

\section{INTRODUCTION}

Apart from being formally correct, a good lattice action should satisfy several requirements: (i) it should induce small cutoff effects in static quantities, (ii) it should have a continuum-like dispersion relation, and (iii) it should be cheap to simulate. Unfortunately, at least in the fermion sector, these requirements tend to be in conflict with each other. For instance, the classic Wilson action [1,2] is good on (iii), but not so much on the first two points. By contrast, an overlap action [3,4] with this operator as a kernel is significantly better on (i), but worse on the remaining two points.

In the literature, there are two main avenues for obtaining a better fermion discretization. The "bottom-up" approach is to expand physical quantities in powers of the lattice spacing $a$, and to demand that the leading cutoff effects are proportional to $\alpha^{n} a$ (or even $a^{2}$ ), where $\alpha$ is the strong coupling constant and $n$ some power. This program of perturbative or nonperturbative $O(a)$-improvement has been carried out successfully [5-9]. The "top-down" approach starts from the concept of a perfect action with zero cutoff effects, perfect chiral symmetry in the sense of the Ginsparg-Wilson relation [10], and a continuum-like dispersion relation. To realize these goals exactly, a Dirac operator $D(x, y)$ is needed with nonzero entry for each $(x, y)$ pair, which is in strong conflict with criterion (iii) above.

In practice, one would like to maintain some degree of sparsity, that is to have an operator which is zero whenever $x$ and $y$ are further apart than a certain threshold. In the literature, the most prominent attempts to realize ultralocal approximate derivatives of the ideal perfect action go by the name truncated perfect action [11-13], hypercube action [14-16], and chirally improved action [17,18]. They differ by the extent through which they make use of the full Dirac-Clifford algebra (the "continuum" operator uses only $\gamma_{\mu}$ with $\mu=1 \ldots 4$ and the identity), and by the criteria used to pin down the various coefficients.
In this article, we pursue a similar approach, albeit with a different focus of which properties should be optimized. We do not attempt to reduce $O\left(a^{2}\right)$ cutoff effects or the amount of chiral symmetry breaking, since it is known how one can get rid of these effects by adding local improvement terms and/or using the overlap recipe. By contrast, we strive for good overall appearance of the eigenvalue spectrum of $D$, and for a continuum-like dispersion relation, because for these properties no systematic improvement scheme is known.

To ease the discussion, let us consider the improved Wilson ("clover") Dirac operator

$$
\begin{aligned}
D(x, y)= & \frac{1}{2} \sum_{\mu}\left\{\left(\gamma_{\mu}-I\right) U_{\mu}(x) \delta_{x+\hat{\mu}, y}-\left(\gamma_{\mu}+I\right) U_{\mu}^{\dagger}(x-\hat{\mu})\right. \\
& \left.\times \delta_{x-\hat{\mu}, y}\right\}+\frac{1}{2 \kappa} \delta_{x, y}-\frac{c_{\mathrm{SW}}}{2} \sum_{\mu<\nu} \sigma_{\mu \nu} F_{\mu \nu} \delta_{x, y},
\end{aligned}
$$

with $\sigma_{\mu \nu}=\frac{\mathrm{i}}{2}\left[\gamma_{\mu}, \gamma_{\nu}\right]$ and $F_{\mu \nu}$ the Hermitean clover-leaf field-strength tensor. In the wavy brackets, there is a discrete Laplacian whose job is to lift 15 out of the 16 species, such that the resulting operator is doubler-free. In other words, the structure of the Wilson operator is

$$
\begin{aligned}
D(x, y)= & \sum_{\mu} \gamma_{\mu} \nabla_{\mu}^{\mathrm{std}}(x, y)-\frac{a}{2} \triangle^{\mathrm{std}}(x, y)+m_{0} \delta_{x, y} \\
& + \text { improvement term, }
\end{aligned}
$$

where $\nabla_{\mu}^{\text {std }}$ denotes the forward-backward symmetric covariant derivative with a 2-point stencil, and $\triangle^{\text {std }}$ the standard covariant Laplacian with a 9-point stencil. The mass parameters in the two representations above relate through $1 /(2 \kappa)=4+a m_{0}$.

The idea explored in this paper is to start from (1), and to replace the covariant derivative $\nabla_{\mu}^{\text {std }}$ and the Laplacian $\triangle^{\text {std }}$ by similar discretizations with improved properties. As a tribute to criterion (iii) above, we shall include only one adjacent layer in each positive or negative direction. Accordingly, both stencils have support on at most $3^{d}$ 
points in $d$ space-time dimensions (which, in the following, will be referred to as 2D, 3D, 4D for $d=2,3,4$, respectively). The choice of the final operator is based on a Darwinistic selection rule. Both for $\nabla_{\mu}$ and $\triangle$, a few varieties with distinct properties are considered, and for each combination the resulting Dirac operator is implemented. Based on the respective eigenvalue spectra and free field dispersion relations, we select the most promising combination in 2D (Sec. II), 3D (Sec. III), and 4D (Sec. IV). Fortunately, it turns out that one choice fares best regarding either criterion, and this choice is the same in any dimension. The resulting operator has no tunable parameters and maintains the property of $\gamma_{5}$-Hermiticity, i.e., $\gamma_{5} D \gamma_{5}=D^{\dagger}$. Details of our implementation, including the overall link-smearing strategy, the gauge covariant derivatives (based on a summation over all shortest paths with backprojection to the group) and tree-level clover improvement, are specified in Sec. V. Practical tests in quenched QCD, with a focus on scaling studies of simple quantities and in comparison to an analogously defined link-smeared tree-level clover improved Wilson operator, are reported in Sec. VI. In Sec. VII, we explore the suitability of our Brillouin operator as a kernel to the overlap procedure, finding a noticeable reduction of the condition number of the shifted Hermitean kernel, and a significant improvement of the locality of the resulting overlap operator. A summary of our findings is given in Sec. VIII, and details of all stencils, both in position and momentum space, are arranged in four appendices with the hope that they might prove useful in applications beyond lattice QCD.

\section{CONSTRUCTION AND MAIN FEATURES IN 2D}

\section{A. Summary of 2D Laplace stencils}

The "standard" stencil of the Laplacian in 2D and the "tilted" variety (as defined in Appendix A) have the Fourier space representation (with $k_{i}=a p_{i}$ the dimensionless wave number)

$$
\begin{gathered}
a^{2} \hat{\triangle}^{\mathrm{std}}\left(k_{1}, k_{2}\right)=2 \cos \left(k_{1}\right)+2 \cos \left(k_{2}\right)-4 \\
=-4 \sin ^{2}\left(k_{1} / 2\right)-4 \sin ^{2}\left(k_{2} / 2\right) \\
a^{2} \hat{\triangle}^{\mathrm{til}}\left(k_{1}, k_{2}\right)=2 \cos \left(k_{1}\right) \cos \left(k_{2}\right)-2 \\
=8 \cos ^{2}\left(k_{1} / 2\right) \cos ^{2}\left(k_{2} / 2\right)-4 \cos ^{2}\left(k_{1} / 2\right) \\
-4 \cos ^{2}\left(k_{2} / 2\right),
\end{gathered}
$$

respectively. From the stencil notation in Appendix A, it is easy to see that in position space the standard Laplacian (2) has only 1-hop contributions (apart from the center element), while the tilted Laplacian (3) has only support at the edge of the $3^{2}$-point area around the center. Both of them discretize the continuum Laplacian in the sense that they deviate from the continuum behavior $\hat{\triangle}^{\text {con }}=-p_{1}^{2}-p_{2}^{2}$ through $O\left(a^{2}\right)$-suppressed terms. Note, however, that the tilted version (3) differs from the standard variety (2) by having a second zero at the edge of the Brillouin zone, i.e., at $k_{1}=k_{2}=\pi$ (in the convention where the Brillouin zone ranges from $-\pi / a$ to $\pi / a$ in every direction).

In 2D, these two stencils form a basis of all Laplace filters with (at most) a 9-point stencil. By taking a linear combination $\hat{\triangle}\left(k_{1}, k_{2}\right)=\alpha \hat{\triangle}^{\text {std }}\left(k_{1}, k_{2}\right)+(1-\alpha) \times$ $\hat{\triangle}^{\text {til }}\left(k_{1}, k_{2}\right)$, one may try to improve certain properties of the discretized Laplacian. In particular, reducing the breaking of the rotational symmetry of the continuum operator is important. Two choices of $\alpha$ are popular in the literature. First, $\alpha=1 / 2$ leads to (what we call) the "Brillouin" filter [19]

$$
\begin{aligned}
a^{2} \hat{\triangle}^{\mathrm{bri}}\left(k_{1}, k_{2}\right) & =\cos \left(k_{1}\right) \cos \left(k_{2}\right)+\cos \left(k_{1}\right)+\cos \left(k_{2}\right)-3 \\
& =4 \cos ^{2}\left(k_{1} / 2\right) \cos ^{2}\left(k_{2} / 2\right)-4
\end{aligned}
$$

since $a^{2} \hat{\triangle}^{\text {bri }}\left(k_{1}, k_{2}\right)=-4$ whenever one of the momenta is $\pm \pi / a$. In other words, $a^{2} \hat{\triangle}^{\text {bri }}$ takes a constant value on the entire boundary of the Brillouin zone. Second, the choice $\alpha=2 / 3$ yields the "isotropic" filter or stencil (see [20] and Refs. [6-7] therein)

$$
\begin{aligned}
a^{2} \hat{\triangle}^{\text {iso }}\left(k_{1}, k_{2}\right)= & {\left[2 \cos \left(k_{1}\right) \cos \left(k_{2}\right)+4 \cos \left(k_{1}\right)\right.} \\
& \left.+4 \cos \left(k_{2}\right)-10\right] / 3 \\
= & {\left[8 \cos ^{2}\left(k_{1} / 2\right) \cos ^{2}\left(k_{2} / 2\right)+4 \cos ^{2}\left(k_{1} / 2\right)\right.} \\
& \left.+4 \cos ^{2}\left(k_{2} / 2\right)-16\right] / 3
\end{aligned}
$$

since for small momenta $a^{2} \hat{\triangle}^{\text {iso }}\left(k_{1}, k_{2}\right)=-a^{2}\left[p_{1}^{2}+p_{2}^{2}\right]+$ $a^{4}\left[p_{1}^{2}+p_{2}^{2}\right]^{2} / 12+O\left(a^{6}\right)$ has $O\left(a^{4}\right)$ terms which depend only on the combination $p_{1}^{2}+p_{2}^{2}$. Put differently, the continuum relation $\hat{\triangle}^{\text {con }}=-p_{1}^{2}-p_{2}^{2}$ is violated on axis (to this order) in the same manner as off axis. Note that this improvement strategy differs from the usual one, where one tries to remove $O\left(a^{n}\right)$ terms, for ever larger $n$, along the axes only.

In Fig. 1, the momentum-space representation of the four Laplacians is shown as a mesh plot (left) and as a contour plot (right). We choose a $24^{2}$ lattice and arrange the center of the Brillouin zone $(p=0)$ in the center of the frame, i.e., the boundaries correspond to momenta $p= \pm \pi / a$. The standard Laplacian that appears in the Wilson operator has a zero in the center and decreases quadratically as one moves away from this point. As we follow the boundary of the Brillouin zone, it oscillates between -4 and -8 . The tilted Laplacian is rather different, since it shows a second zero at $p=(\pi / a, \pi / a)$, a quarter of which is seen in each corner. The Brillouin Laplacian has just one zero and achieves complete flatness at the boundary of the Brillouin zone. Finally, the isotropic Laplacian achieves best isotropy near the center of the Brillouin zone, meaning that one can move relatively far 

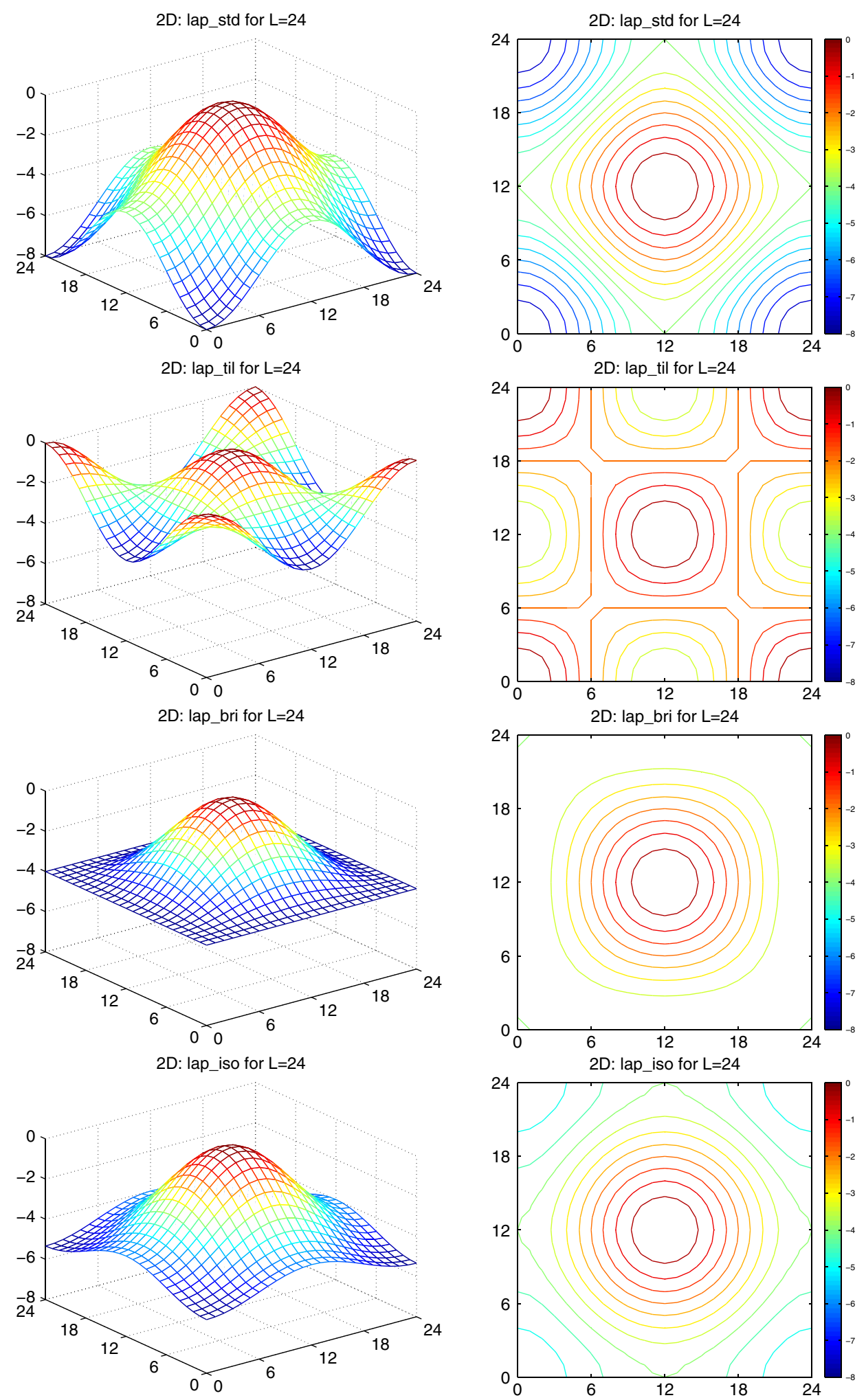

FIG. 1 (color online). Fourier transformation of the four Laplace stencils considered in 2D.

out from the center until its equipotential lines become noticeably noncircular.

In Fig. 2, the momentum-space representations of the three derivatives specified in Appendix A are shown as a mesh plot (left) and as a contour plot (right). The standard derivative that appears in the Wilson operator is a pure $\sin \left(p_{1} / a\right)$, without any structure in the transverse direction. The Brillouin derivative modulates the transverse direction 
2D: der std for $\mathrm{L}=24$

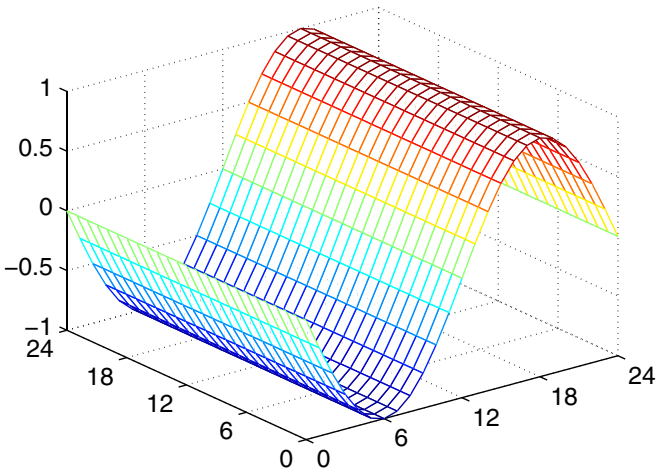

2D: der_bri for $L=24$

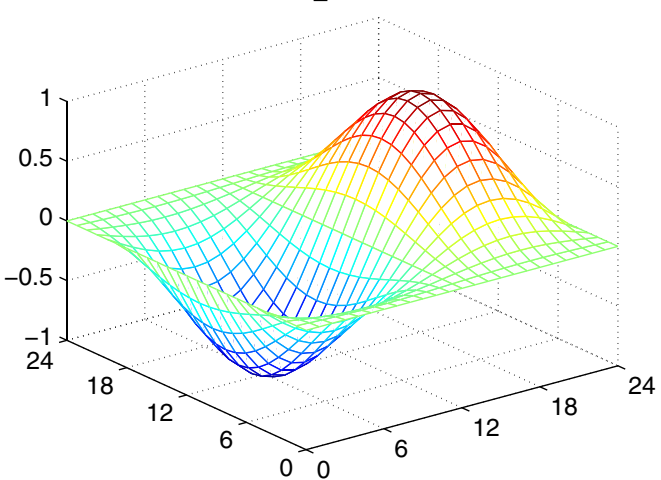

2D: der_iso for $L=24$

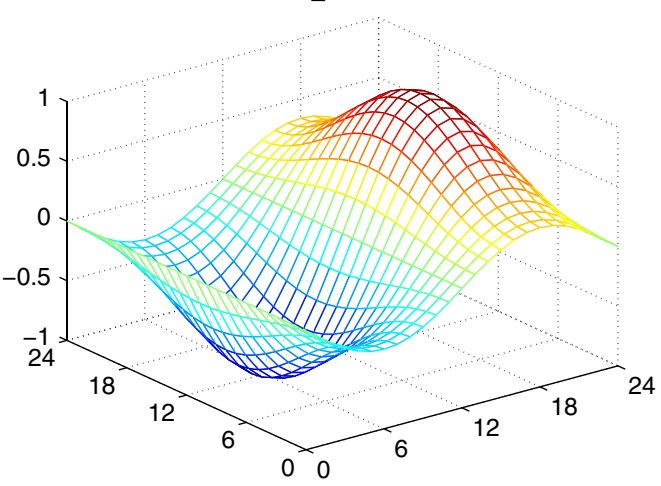

D: der std for $L=24$
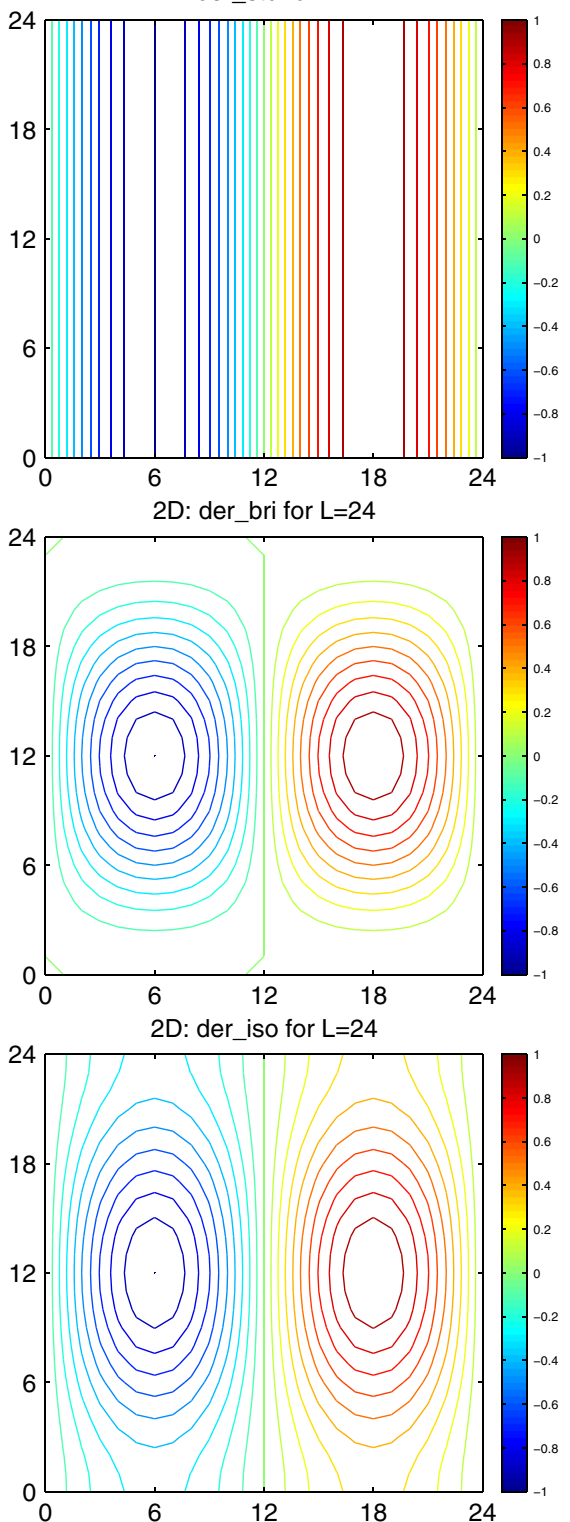

FIG. 2 (color online). Fourier transformation of the three derivative stencils considered in 2D.

to the point that a strict zero is realized on the entire boundary. The isotropic operator modulates the transverse direction in a less pronounced manner. For the reasons behind the name of this latter operator, which may sound a bit paradoxical, see Appendix D.

\section{B. Eigenvalue spectra in 2D}

Given the four choices of $\Delta$ discussed above and the three choices of $\nabla_{\mu}$, we can construct 12 Dirac operators and study their eigenvalue spectra. As the gauge group is irrelevant in this step, we prepare a thermalized background in the $U(1)$ gauge theory with $L / a=24$ at $\beta=3.3$.

In Fig. 3, the eigenvalue spectra of the 12 operators without improvement $\left(c_{\mathrm{SW}}=0\right)$ are shown. The (type of)
Laplacian is used as the row index of the panel and the derivative as the column index. Out of these 12 constructions, 9 are undoubled fermion operators, while 3 yield two species in the continuum limit. Let us discuss the undoubled operators first. The three operators with $\triangle^{\text {std }}$ have three branches, the left-most physical branch with the correct sensitivity to the topological charge of the gauge background, a doubly populated branch of wrong-chirality doublers near $\operatorname{Re}(z)=2$, and another species with the correct chirality near $\operatorname{Re}(z)=4$. Here the choice of derivative affects the spreading of the unphysical branches in the imaginary direction, but it leaves the topological properties of the spectrum unaffected. The three operators with $\triangle^{\text {bri }}$ have only two branches, the left (physical) one is undoubled with the correct chirality, the right one includes three 

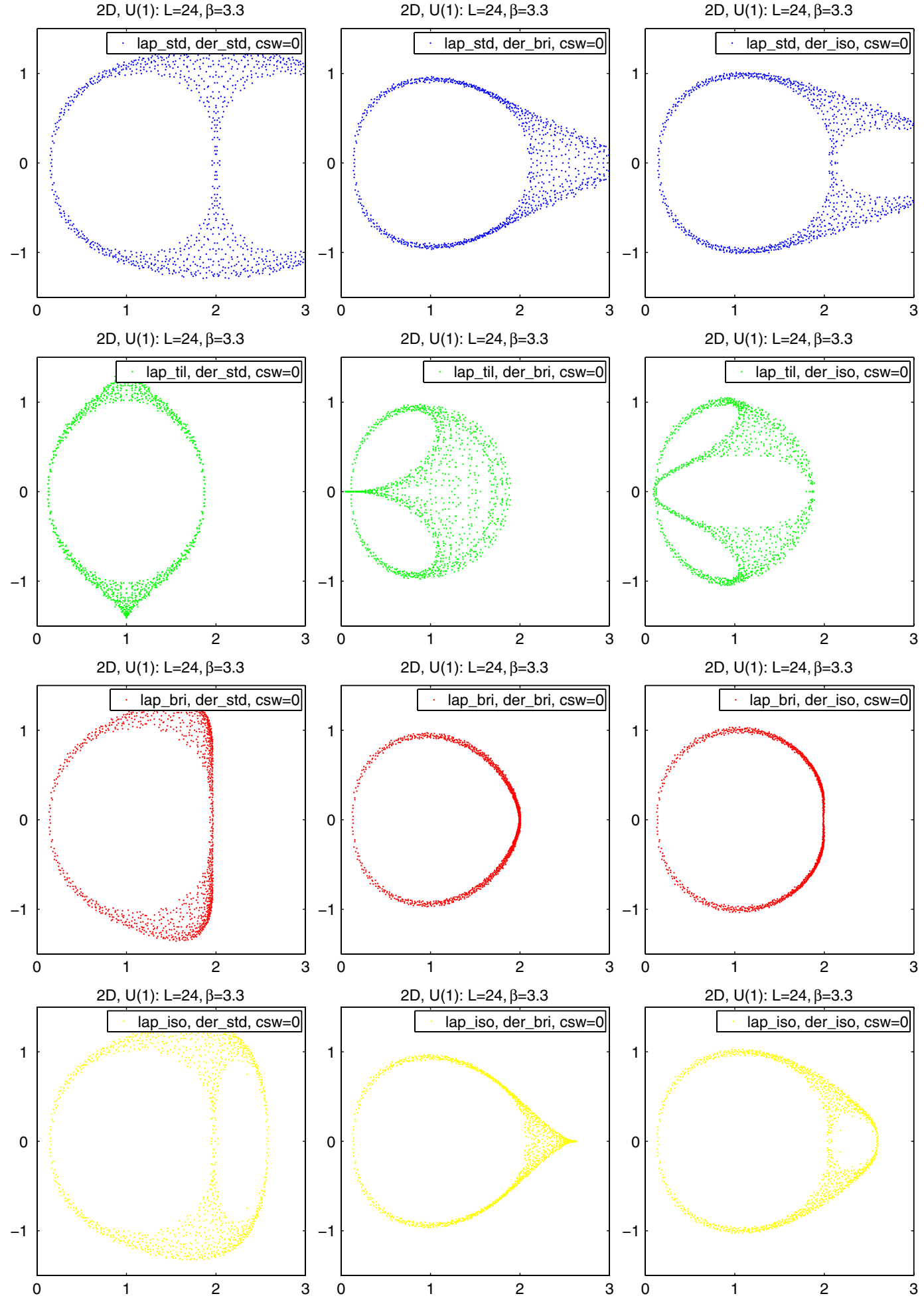

FIG. 3 (color online). Eigenvalue spectra of all operators considered in $2 \mathrm{D}$ with $c_{\mathrm{SW}}=0$.

species, two with the wrong chirality and one with the correct chirality. The three operators with $\triangle^{\text {iso }}$ have spectra which resemble those in the first row, except that the lifting of the last branch is reduced, in perfect agreement with what one expects on the basis of Fig. 1. The most interesting spectra from a field theoretical point of view are those of the operators with $\triangle^{\text {til }}$. Naively, one would expect that they yield a legal 2-flavor operator (in 2D), as the second row of Fig. 1 shows that this Laplacian has two zeros. With the naive derivative operator employed, this expectation 

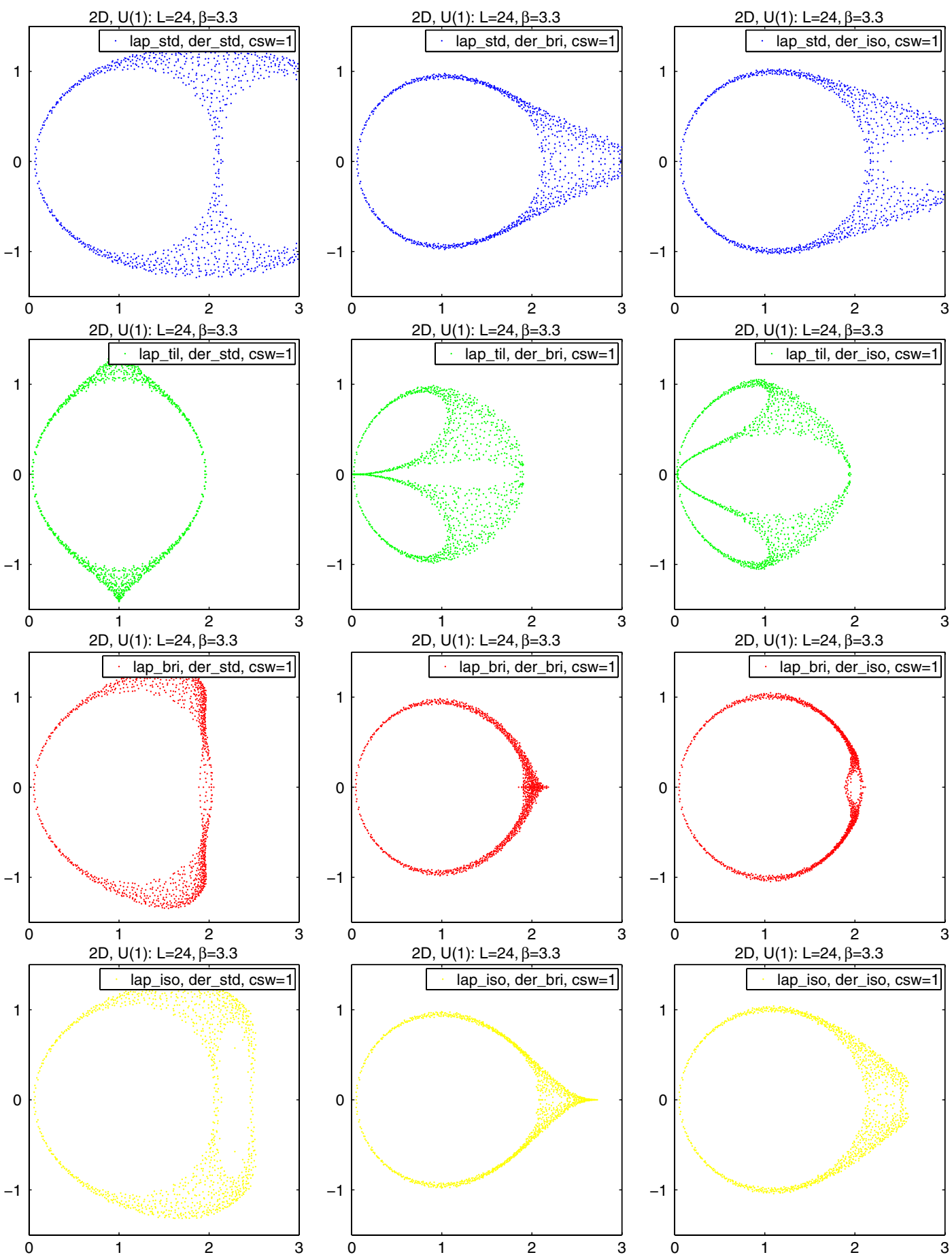

FIG. 4 (color online). Eigenvalue spectra of all operators considered in $2 \mathrm{D}$ with $c_{\mathrm{SW}}=1$.

happens to be correct; the resulting operator has two equal chirality species, ${ }^{1}$ which survive in the continuum limit and two doublers (again with equal, but this time wrong chirality) which decouple in the continuum limit. With any of the two remaining derivatives employed, things are a bit

\footnotetext{
${ }^{1}$ Note the difference to staggered fermions in $2 \mathrm{D}$, where the two species have opposite chiralities.
}

more involved, as the "thorn" or the "bump" in the middle or right panel of the second row illustrates. The point is that there is an interference ${ }^{2}$ between the dimension 5 Laplacian and the dimension 4 derivative; the "cross talk" phenomena in the 2 nd and 3rd column of the second row exemplify that

\footnotetext{
${ }^{2}$ We avoid the word "mixing", because this is a phenomenon which persists in the weak coupling limit.
} 

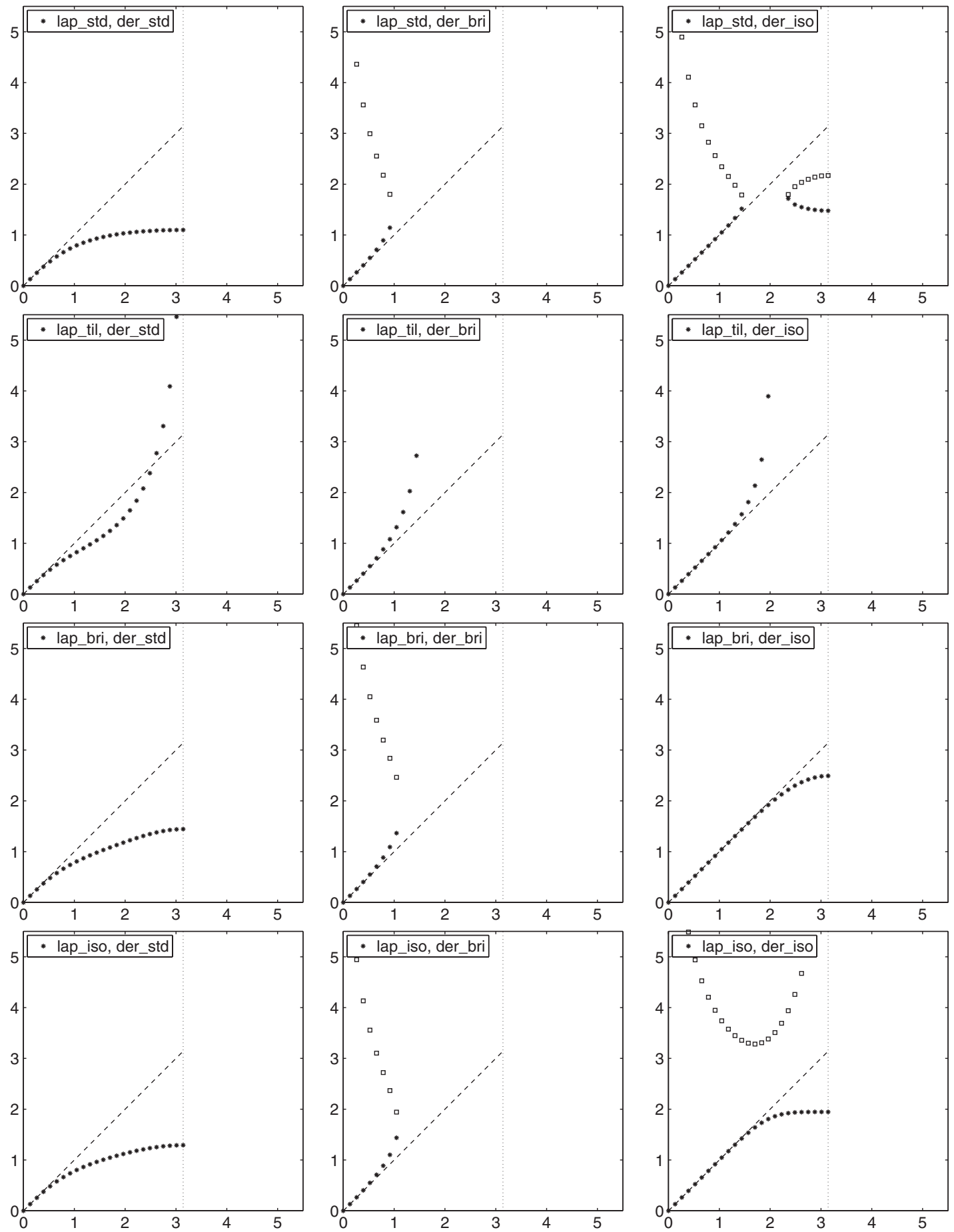

FIG. 5. Free-field dispersion relations of all operators considered in $2 \mathrm{D}$, where $|p|_{\max }=\pi / a$.

this may affect the structural properties of the fermion operator. In either case, one of the would-be physical species fails to cling nicely on the imaginary axis for small momenta. While the operator in the 2 nd column is clearly not a legal discretization (the thorn violates the property $D \sim$ i $p_{\mu} \gamma_{\mu}$ ), the version in the 3rd column may represent a legal 2-flavor discretization of the Dirac operator (though, likely, with terrible cutoff effects). In summary, from this first overview it appears that the four operators towards the lower right corner of this figure seem to be the most promising.

In Fig. 4, the same survey is repeated with tree-level improvement $\left(c_{\mathrm{SW}}=1\right.$, cf. Sec. VC). Relative to the previous figure, changes seem to be mild. However, an interesting point is that the clover term shifts correct-type chirality branches (slightly) to the left and wrong-kind chirality branches (slightly) to the right. As a result, for 
the 9 undoubled operators the additive mass renormalization (the offset of the physical branch at zero imaginary part) is always reduced. Also, our statements in the previous paragraph regarding the chiralities of the unphysical branches can now be checked, because they map into a prediction of the effect of the clover term. At this point we can probably say that $\left(\triangle^{\text {bri }}, \nabla^{\text {iso }}\right)$ fares best in the sense that its eigenvalue spectrum is closest to that of an operator satisfying the Ginsparg-Wilson relation. ${ }^{3}$

\section{Free-field dispersion relations in 2D}

As mentioned in the Introduction, the free-field dispersion relation of the fermion operator is of utmost importance, as this is a property for which there is no systematic improvement scheme (apart from taking the continuum limit). With standard $\gamma$-matrix identities, it follows that the inverse of $D=\sum \gamma_{\mu} \nabla_{\mu}-\frac{r}{2} \Delta+m$ is given by $D^{-1}=$ $\left(-\sum \gamma_{\mu} \nabla_{\mu}-\frac{r}{2} \triangle+m\right) /\left(\left[\frac{r}{2} \triangle-m\right]^{2}-\sum \nabla_{\mu}^{2}\right)$, where $r$ is the Wilson parameter. Accordingly, to work out the dispersion relation, we have to search for zeros of $\left[\frac{r}{2} \Delta-m\right]^{2}-$ $\sum \nabla_{\mu}^{2}$, where $\triangle$ and $\nabla$ denote any one of the Laplacians or derivatives introduced above.

In Fig. 5, we show, for each operator, the real solutions for $r=1$ and $m=0$ over half the Brillouin zone on a 2D lattice with $L / a=48$. The dispersion relation of the standard Wilson operator $\left(\triangle^{\text {std }}, \nabla^{\text {std }}\right)$ deviates soon from the dashed line, which corresponds to the continuum dispersion relation; in particular, towards the boundary of the Brillouin zone the distortion is significant. Black boxes indicate a second real solution. If sufficiently high, this is harmless, as this branch decouples in the continuum limit. Note that (in certain parts of the Brillouin zone) some operators have only complex roots. While this proves, again, irrelevant in the continuum limit, it is certainly not a desirable feature. Overall, it is clear that the combination $\left(\triangle^{\text {bri }}, \nabla^{\text {iso }}\right)$ fares best in the sense that its dispersion relation is closest to the one in the continuum.

\section{CONSTRUCTION AND MAIN FEATURES IN 3D}

\section{A. Summary of 3D Laplace stencils}

The standard stencil of the Laplacian in 3D and the tilted variety (as defined in Appendix B) have the Fourier space representation

$$
\begin{aligned}
a^{2} \hat{\triangle}^{\mathrm{std}}\left(k_{1}, k_{2}, k_{3}\right)= & 2 \cos \left(k_{1}\right)+2 \cos \left(k_{2}\right)+2 \cos \left(k_{3}\right)-6 \\
= & -4 \sin ^{2}\left(k_{1} / 2\right)-4 \sin ^{2}\left(k_{2} / 2\right) \\
& -4 \sin ^{2}\left(k_{3} / 2\right)
\end{aligned}
$$

\footnotetext{
${ }^{3}$ The eigenvalue spectrum of such an operator is in the unit circle centered at point 1 on the real axis [10].
}

$$
\begin{aligned}
a^{2} \hat{\triangle}^{\mathrm{til}}\left(k_{1}, k_{2}, k_{3}\right)= & 2 \cos \left(k_{1}\right) \cos \left(k_{2}\right) \cos \left(k_{3}\right)-2 \\
= & 16 \cos ^{2}\left(k_{1} / 2\right) \cos ^{2}\left(k_{2} / 2\right) \cos ^{2}\left(k_{3} / 2\right) \\
& -8 \cos ^{2}\left(k_{1} / 2\right) \cos ^{2}\left(k_{2} / 2\right)-\ldots \\
& +4 \cos ^{2}\left(k_{1} / 2\right)+\ldots-4,
\end{aligned}
$$

respectively, with the ellipses denoting cyclic permutations. From the stencil notation in Appendix B, it is easy to see that the former has only 1-hop contributions, while the latter has only 3-hop contributions (apart from the central element). For asymptotically small momenta they both reduce to the continuum relation $\hat{\triangle}=p_{1}^{2}+p_{2}^{2}+p_{3}^{2}$, but the tilted stencil has three additional zeros at the boundary of the Brillouin zone $\left[\hat{\Delta}^{\text {til }}\right.$ vanishes at $\left(k_{1}, k_{2}, k_{3}\right)-(\pi, \pi, \pi) / 2=( \pm \pi, \pm \pi, \pm \pi) / 2$ with an odd number of minus signs].

In 3D the discretizations of the continuum Laplacian which are analogous to (4) and (5) in 2D are no longer simple linear combinations of (6) and (7), because one could come up with a Laplacian which has only 2-hop contributions (apart from the center element). They read

$$
\begin{aligned}
a^{2} \hat{\triangle}^{\mathrm{bri}}\left(k_{1}, k_{2}, k_{3}\right)= & {\left[\cos \left(k_{1}\right) \cos \left(k_{2}\right) \cos \left(k_{3}\right)+\cos \left(k_{1}\right)\right.} \\
& \left.\times \cos \left(k_{2}\right)+\ldots+\cos \left(k_{1}\right)+\ldots-7\right] / 2 \\
= & 4 \cos ^{2}\left(k_{1} / 2\right) \cos ^{2}\left(k_{2} / 2\right) \cos ^{2}\left(k_{3} / 2\right)-4
\end{aligned}
$$

$$
\begin{aligned}
a^{2} \hat{\triangle}^{\text {iso }}\left(k_{1}, k_{2}, k_{3}\right)= & {\left[\cos \left(k_{1}\right) \cos \left(k_{2}\right) \cos \left(k_{3}\right)+3 \cos \left(k_{1}\right)\right.} \\
& \left.\times \cos \left(k_{2}\right)+\ldots+5 \cos \left(k_{1}\right)+\ldots-25\right] / 6 \\
= & {\left[4 \cos ^{2}\left(k_{1} / 2\right) \cos ^{2}\left(k_{2} / 2\right) \cos ^{2}\left(k_{3} / 2\right)\right.} \\
& \left.+4 \cos ^{2}\left(k_{1} / 2\right) \cos ^{2}\left(k_{2} / 2\right)+\ldots-16\right] / 3,
\end{aligned}
$$

respectively, and their distinctive features are as follows. The Brillouin Laplacian (8) takes a constant value on the entire boundary of the Brillouin zone, since $a^{2} \hat{\triangle}^{\text {bri }}\left(k_{1}, k_{2}, k_{3}\right)=-4$ whenever one of the momenta is $\pm \pi / a$. On the other hand, the Laplacian (9) is called isotropic since $a^{2} \hat{\triangle}^{\text {iso }}\left(k_{1}, k_{2}, k_{3}\right)=-a^{2}\left[k_{1}^{2}+k_{2}^{2}+k_{3}^{2}\right]+$ $a^{4}\left[k_{1}^{2}+k_{2}^{2}+k_{3}^{2}\right]^{2} / 12+O\left(a^{6}\right)$ has $O\left(a^{4}\right)$ terms which depend only on the combination $k_{1}^{2}+k_{2}^{2}+k_{3}^{2}$. In other words, $\hat{\triangle}^{\text {iso }}\left(k_{1}, k_{2}, k_{3}\right)$ respects rotational symmetry even in the leading term through which it deviates from the continuum.

In 3D there are 3 linearly independent Laplacians with (at most) a 27-point stencil, and any 3 out of the 4 elements (6)-(9) form a basis. A systematic treatment is given in [21].

\section{B. Eigenvalue spectra in 3D}

Like in the preceding section, with four options for $\triangle$ and three for $\nabla$, we can construct 12 Dirac operators and 
study their eigenvalue spectra. As the gauge group is irrelevant in this step, we prepare a thermalized background in the $U(1)$ gauge theory with $L / a=12$ at $\beta=2$.2. A point worth mentioning is that in odd dimensions there is an ambiguity regarding the representation of the $\gamma$-matrices
[22]; we opt for the 4-dimensional representation (the same one that we will use in 4D).

In Fig. 6, the eigenvalues of the 12 operators without improvement $\left(c_{\mathrm{SW}}=0\right)$ are shown. This time we refrain from showing the counterpart with improvement, as the
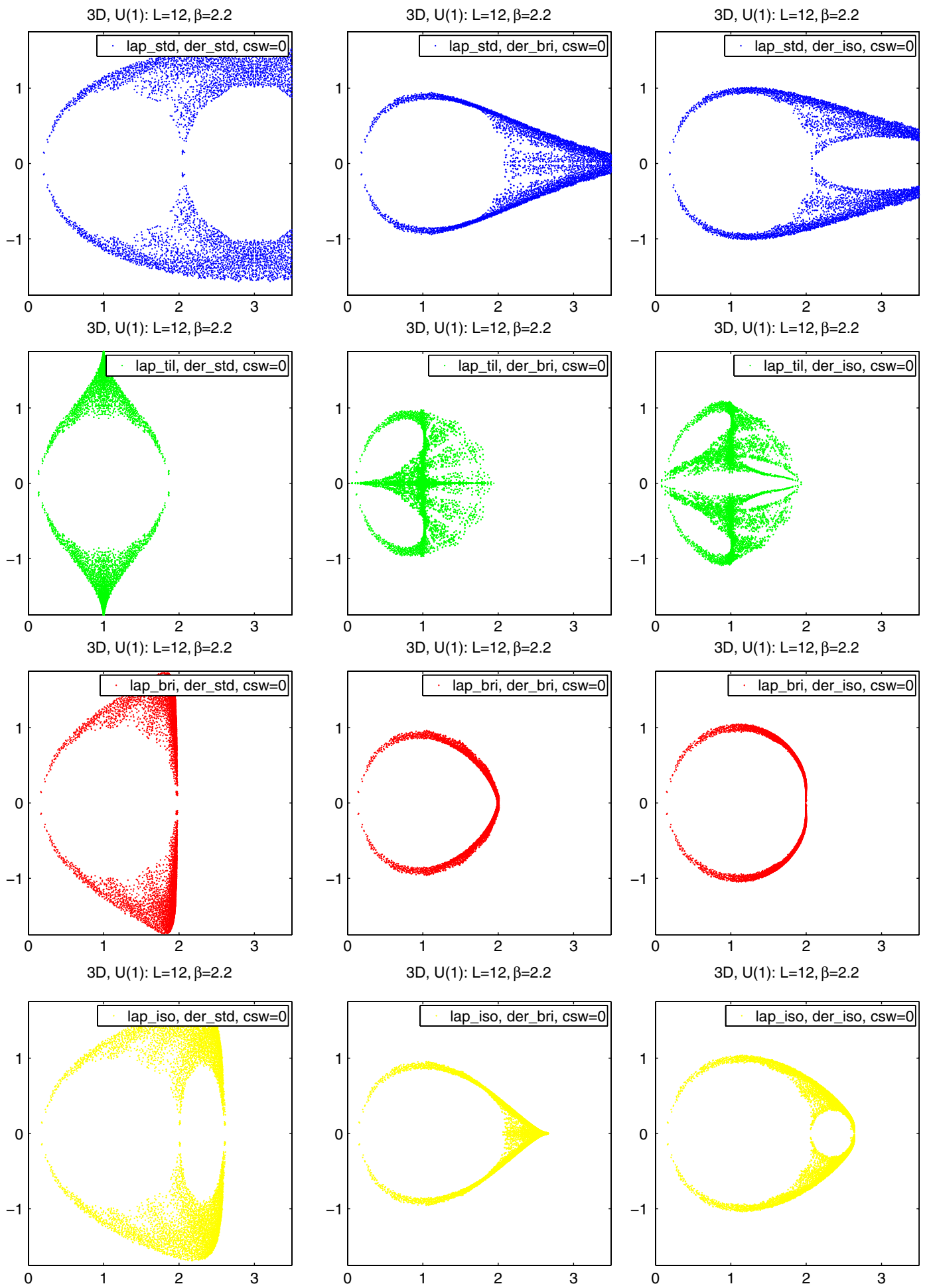

FIG. 6 (color online). Eigenvalue spectra of all operators considered in 3D with $c_{\mathrm{SW}}=0$. 

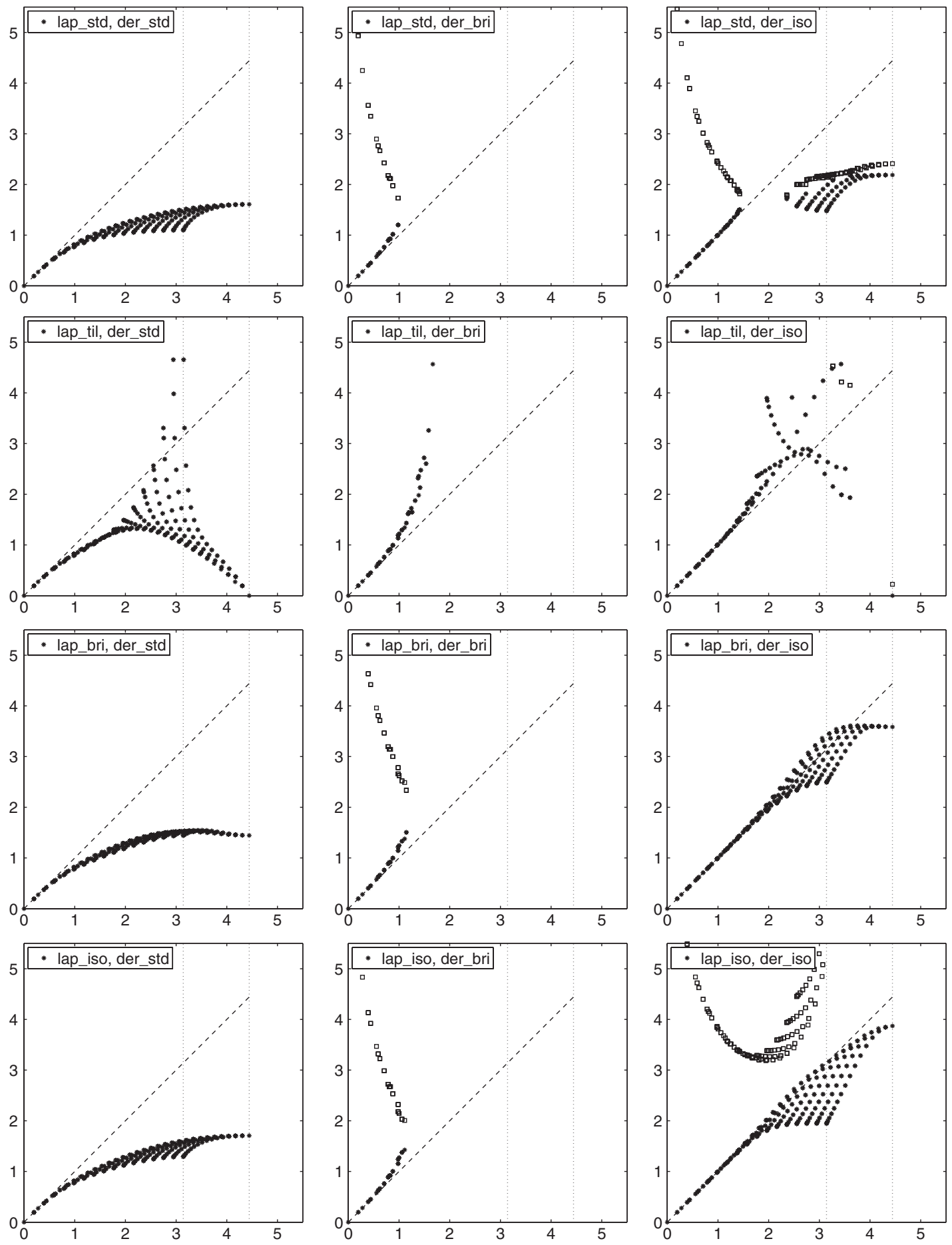

FIG. 7. Free-field dispersion relations of all operators considered in $3 \mathrm{D}$, where $|p|_{\max }=\sqrt{2} \pi / a$.

difference is (again) minor. Just as in the previous section, the Laplacian features are shown as the row index of the panel, and the derivative as the column index. Out of these 12 constructions, 9 are undoubled fermion operators, while 3 yield four species in the continuum limit. The gross features of most operators are rather similar to those in the 2D case, which was discussed in great detail above. Perhaps the most significant difference is that the operators with the standard Laplacian (first row) have branches at $\operatorname{Re}(z) \simeq 0,2,4,6$, with multiplicities $1,3,3$, 1, respectively. If the standard Laplacian is replaced by the Brillouin Laplacian (third row) or the isotropic Laplacian (fourth row), the doublers are lifted more equally; in particular, the former alternative arranges them all near $\operatorname{Re}(z) \simeq 2$. With the tilted Laplacian and the standard derivative, the 8 species arrange themselves in groups of 4 at $\operatorname{Re}(z) \simeq 0$ 
and $\operatorname{Re}(z) \simeq 2$, respectively. As soon as the standard derivative is replaced by the Brillouin or isotropic variety, some of the 4 would-be-physical modes cross over to the unphysical side so quickly that the resulting operator is barely usable. Looking at the whole figure, one would say that the combination $\left(\triangle^{\text {bri }}, \nabla^{\text {iso }}\right)$ fares best in the sense that its eigenvalue spectrum is reasonably circular.

\section{Free-field dispersion relations in 3D}

In Fig. 7, we show, for each operator, the real solutions for $r=1$ and $m=0$ over half the Brillouin zone on a 3D lattice with $L / a=32$. The dispersion relation of the standard Wilson operator $\left(\triangle^{\text {std }}, \nabla^{\text {std }}\right)$ deviates soon from the dashed line, which corresponds to the continuum dispersion relation; in particular, towards the boundary of the Brillouin zone the distortion is significant. In 3D the dispersion relation is no longer a simple curve, it depends on the orientation of the spatial momentum. If $\mathbf{p}$ is chosen on axis, the $2 \mathrm{D}$ dispersion relation is reproduced. The latter ends at $\sqrt{2} \pi / a$ and features as an embedding curve to the $3 \mathrm{D}$ dispersion relation which now reaches out to $\sqrt{3} \pi / a$. Again, some operators admit a second real solution (open boxes) which decouples in the continuum, and some operators have, for certain combinations of $\left(p_{1}, p_{2}\right)$, only complex solutions. Overall, it is clear that the combination ( $\triangle^{\text {bri }}, \nabla^{\text {iso }}$ ) fares best in the sense that its dispersion relation is closest to the one in the continuum.

\section{CONSTRUCTION AND MAIN FEATURES IN 4D}

\section{A. Summary of 4D Laplace stencils}

The standard stencil of the Laplacian in 4D and the tilted variety (as defined in Appendix C) have the Fourier space representation

$$
\begin{gathered}
a^{2} \hat{\triangle}^{\mathrm{std}}\left(k_{1}, k_{2}, k_{3}, k_{4}\right)=2 \cos \left(k_{1}\right)+2 \cos \left(k_{2}\right)+2 \cos \left(k_{3}\right)+2 \cos \left(k_{4}\right)-8 \\
=-4 \sin ^{2}\left(k_{1} / 2\right)-4 \sin ^{2}\left(k_{2} / 2\right)-4 \sin ^{2}\left(k_{3} / 2\right)-4 \sin ^{2}\left(k_{4} / 2\right) \\
a^{2} \hat{\triangle}^{\mathrm{til}}\left(k_{1}, k_{2}, k_{3}, k_{4}\right)=2 \cos \left(k_{1}\right) \cos \left(k_{2}\right) \cos \left(k_{3}\right) \cos \left(k_{4}\right)-2 \\
=32 \cos ^{2}\left(k_{1} / 2\right) \cos ^{2}\left(k_{2} / 2\right) \cos ^{2}\left(k_{3} / 2\right) \cos ^{2}\left(k_{4} / 2\right)-16 \cos ^{2}\left(k_{1} / 2\right) \cos ^{2}\left(k_{2} / 2\right) \cos ^{2}\left(k_{3} / 2\right)-\ldots \\
+8 \cos ^{2}\left(k_{1} / 2\right) \cos ^{2}\left(k_{2} / 2\right)+\ldots-4 \cos ^{2}\left(k_{1}\right)-\ldots,
\end{gathered}
$$

respectively, with the ellipses denoting cyclic permutations. From the stencil notation in Appendix $\mathrm{C}$ it is easy to see that the former has only 1-hop contributions, while the latter has only 4-hop contributions (apart from the central element). For asymptotically small momenta, they both reproduce the continuum relation $\hat{\triangle}=p_{1}^{2}+p_{2}^{2}+p_{3}^{2}+p_{4}^{2}$, but the tilted stencil has seven additional zeros at the boundary of the Brillouin zone $\left[\hat{\triangle}^{\text {til }}\right.$ vanishes at $\left(k_{1}, k_{2}, k_{3}, k_{4}\right)-(\pi, \pi, \pi, \pi) / 2=$ $( \pm \pi, \pm \pi, \pm \pi, \pm \pi) / 2$ with an even number of minus signs].

In 4D, the discretizations of the continuum Laplacian, which are analogous to (4) and (5), read

$$
\begin{aligned}
a^{2} \hat{\triangle}^{\mathrm{bri}}\left(k_{1}, k_{2}, k_{3}, k_{4}\right)= & {\left[\cos \left(k_{1}\right) \cos \left(k_{2}\right) \cos \left(k_{3}\right) \cos \left(k_{4}\right)+\cos \left(k_{1}\right) \cos \left(k_{2}\right) \cos \left(k_{3}\right)+\ldots+\cos \left(k_{1}\right) \cos \left(k_{2}\right)\right.} \\
& \left.+\ldots+\cos \left(k_{1}\right)+\ldots-15\right] / 4 \\
= & 4 \cos ^{2}\left(k_{1} / 2\right) \cos ^{2}\left(k_{2} / 2\right) \cos ^{2}\left(k_{3} / 2\right) \cos ^{2}\left(k_{4} / 2\right)-4
\end{aligned}
$$

$$
\begin{aligned}
a^{2} \hat{\triangle}^{\text {iso }}\left(k_{1}, k_{2}, k_{3}, k_{4}\right)= & {\left[2 \cos \left(k_{1}\right) \cos \left(k_{2}\right) \cos \left(k_{3}\right) \cos \left(k_{4}\right)+7 \cos \left(k_{1}\right) \cos \left(k_{2}\right) \cos \left(k_{3}\right)+\ldots+20 \cos \left(k_{1}\right) \cos \left(k_{2}\right)\right.} \\
& \left.+\ldots+25 \cos \left(k_{1}\right)+\ldots-250\right] / 54 \\
= & {\left[16 \cos ^{2}\left(k_{1} / 2\right) \cos ^{2}\left(k_{2} / 2\right) \cos ^{2}\left(k_{3} / 2\right) \cos ^{2}\left(k_{4} / 2\right)+20 \cos ^{2}\left(k_{1} / 2\right) \cos ^{2}\left(k_{2} / 2\right) \cos ^{2}\left(k_{3} / 2\right)\right.} \\
& \left.+\ldots+16 \cos ^{2}\left(k_{1} / 2\right) \cos ^{2}\left(k_{2} / 2\right)+\ldots-16 \cos ^{2}\left(k_{1} / 2\right)-\ldots-128\right] / 27,
\end{aligned}
$$

respectively, and their distinctive features are as follows. The Brillouin Laplacian (12) takes a constant value on the entire boundary of the Brillouin zone, since $a^{2} \hat{\triangle}^{\text {bri }}\left(k_{1}, k_{2}, k_{3}, k_{4}\right)=-4$ whenever one of the momenta is $\pm \pi / a$. On the other hand, the Laplacian (13) is called isotropic since $a^{2} \widehat{\triangle}^{\text {iso }}\left(k_{1}, k_{2}, k_{3}, k_{4}\right)=-a^{2}\left[k_{1}^{2}+k_{2}^{2}+k_{3}^{2}+k_{4}^{2}\right]+a^{4}\left[k_{1}^{2}+k_{2}^{2}+k_{3}^{2}+k_{4}^{2}\right]^{2} /$ $12+O\left(a^{6}\right)$ has $O\left(a^{4}\right)$ terms which depend only on the combination $k_{1}^{2}+k_{2}^{2}+k_{3}^{2}+k_{4}^{2}$. In other words, $\widehat{\triangle}^{\text {iso }}\left(k_{1}, k_{2}, k_{3}, k_{4}\right)$ respects rotational symmetry even in the leading term through which it deviates from the continuum.

In 4D, there are 4 linearly independent Laplacians with (at most) an 81-point stencil, and the 4 elements (10)-(13) form a basis. We are unaware of any systematic treatment in the literature. 


\section{B. Eigenvalue spectra in 4D}

Like in the previous two sections, with four options for $\triangle$ and three for $\nabla$, we can construct 12 Dirac operators and study their eigenvalue spectra. As the gauge group is irrelevant in this step, we prepare a thermalized background in the $U(1)$ gauge theory with $L / a=6$ at $\beta=1.1$.

In Fig. 8, the eigenvalues of the 12 operators without improvement $\left(c_{\mathrm{SW}}=0\right)$ are shown. Again, we refrain from
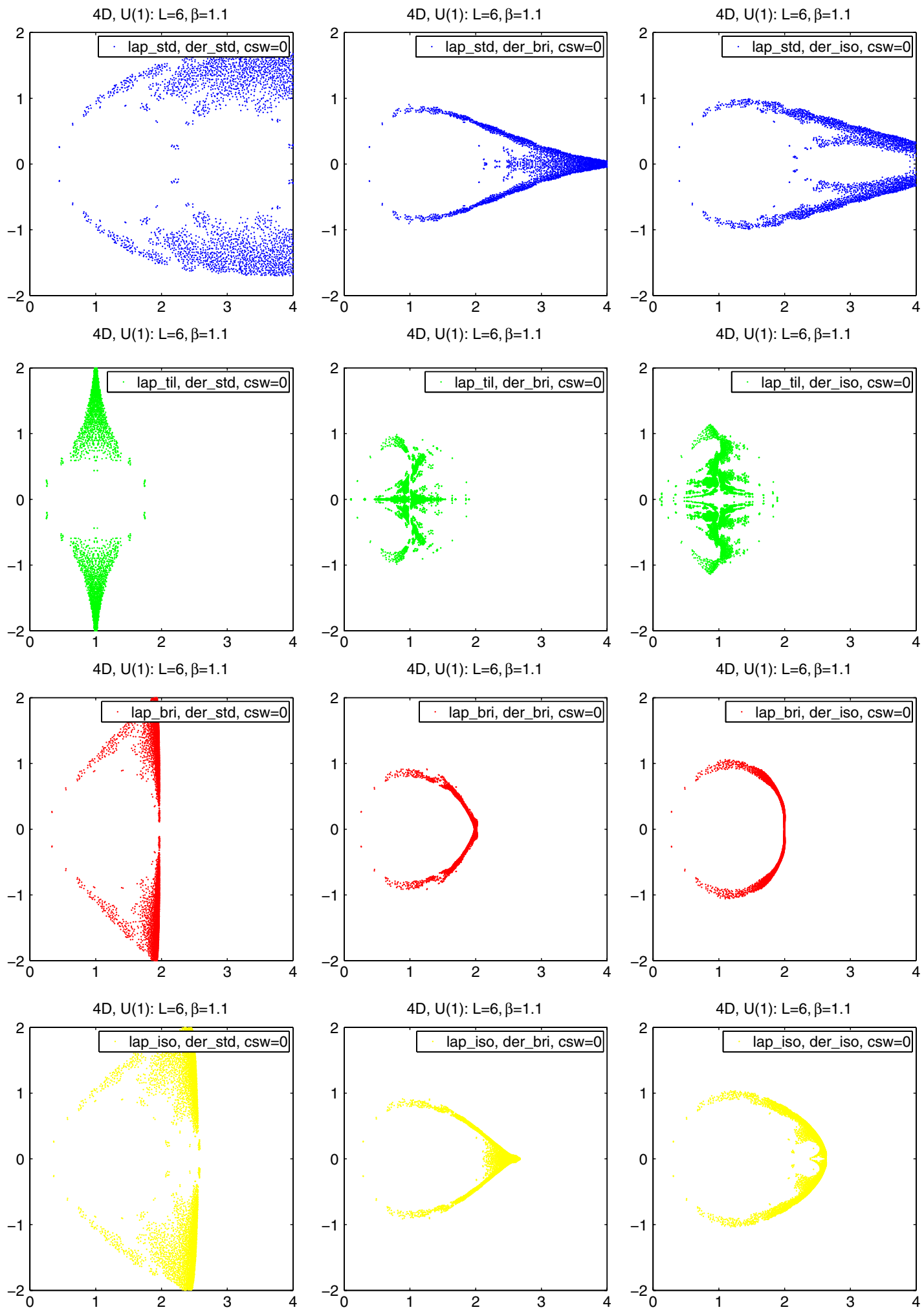

FIG. 8 (color online). Eigenvalue spectra of all operators considered in 4D with $c_{\mathrm{SW}}=0$. 

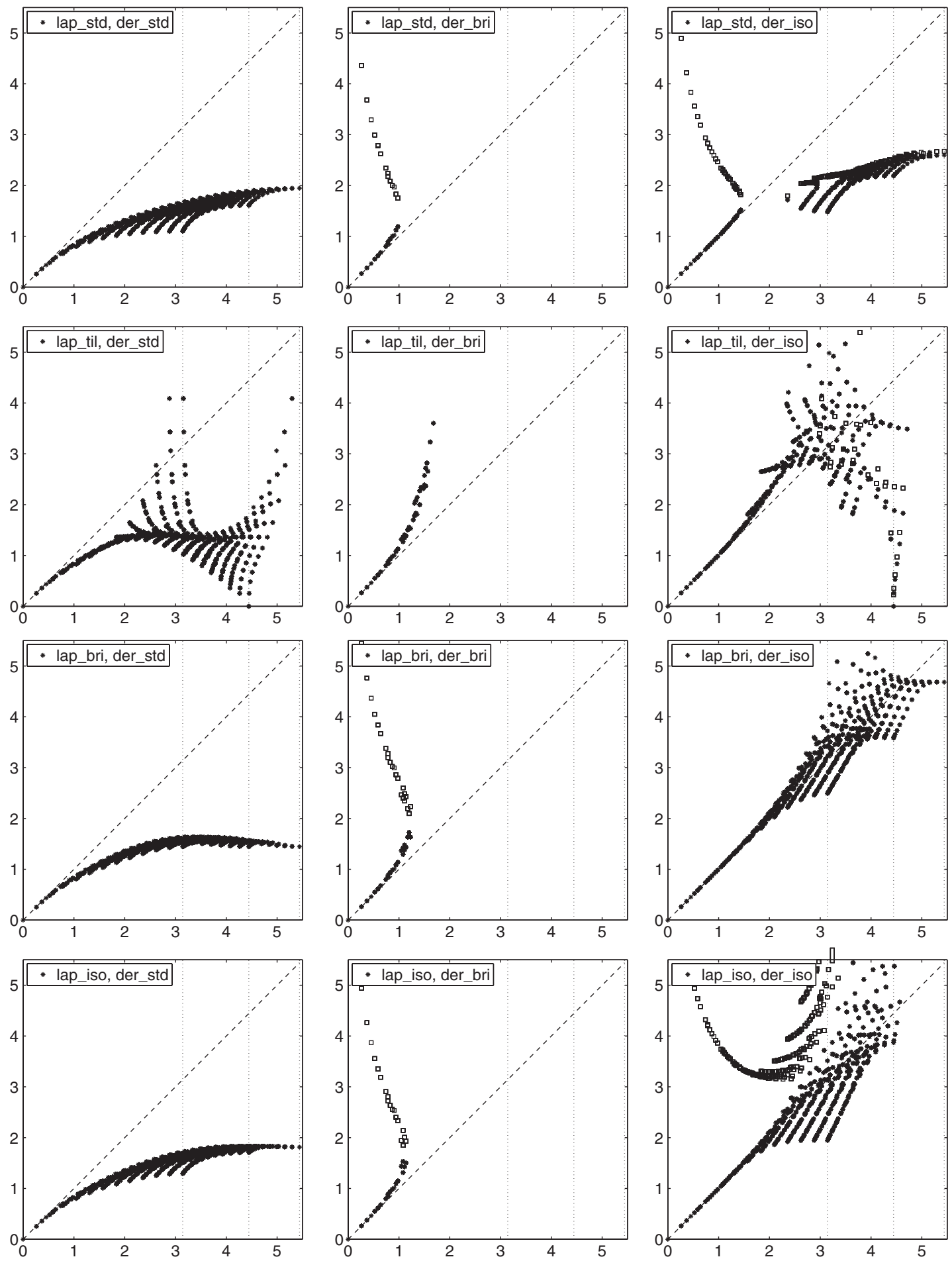

FIG. 9. Free-field dispersion relations of all operators considered in $4 \mathrm{D}$, where $|p|_{\max }=\sqrt{3} \pi / a$.

showing the counterpart with improvement, as the difference is marginal. Following the tradition of the previous sections, the Laplacian features are shown as the row index of the panel, and the derivative as the column index. Out of these 12 constructions, 9 are undoubled fermion operators, while 3 yield eight species in the continuum limit. Again, the gross features of these operators are rather similar to their 2D and 3D counterparts. This time, the operators with the standard Laplacian (first row) have branches at $\operatorname{Re}(z) \simeq$ $0,2,4,6,8$, with multiplicities $1,4,6,4,1$, respectively, and with alternating chiralities. Replacing the standard Laplacian by the Brillouin Laplacian (third row) or the isotropic Laplacian (fourth row), the lifting of the doublers is reduced. With the tilted Laplacian and the standard 
derivative, the 16 species arrange themselves in groups of 8 at $\operatorname{Re}(z) \simeq 0$ (correct-type chirality) and $\operatorname{Re}(z) \simeq 2$ (wrong-kind chirality), respectively. ${ }^{4}$ Once the standard derivative is replaced by the Brillouin or isotropic variety, some cross talk between the marginal and the irrelevant pieces becomes apparent. Looking at the whole figure, one would say that the combination $\left(\triangle^{\text {bri }}, \nabla^{\text {iso }}\right.$ ) fares best in the sense that its eigenvalue spectrum is closest to that of a Ginsparg-Wilson action.

\section{Free-field dispersion relations in 4D}

In Fig. 9, we show, for each operator, the real solutions for $r=1$ and $m=0$ over half the Brillouin zone on a 4D lattice with $L / a=24$. The dispersion relation of the standard Wilson operator $\left(\triangle^{\text {std }}, \nabla^{\text {std }}\right)$ deviates soon from the dashed line, which corresponds to the continuum dispersion relation, and shows large effects of anisotropy. If $\mathbf{p}$ is chosen on-axis, the $2 \mathrm{D}$ dispersion relation is reproduced. If $\mathbf{p}$ is chosen as a multiple of $(1,1,0)$, the $3 \mathrm{D}$ dispersion relation is reproduced. If $\mathbf{p}$ is chosen as a multiple of $(1,1,1)$ entries at the upper border are generated; they go out to $\sqrt{3} \pi / a$. Again, some operators admit a second real solution (open boxes) which decouples in the continuum, and some operators have, for certain combinations of $\left(p_{1}, p_{2}, p_{3}\right)$, only complex solutions. Overall, it is clear that the combination $\left(\triangle^{\text {bri }}, \nabla^{\text {iso }}\right)$ fares best in the sense that its dispersion relation is closest to the one in the continuum.

\section{SPECIFICATION OF OPERATOR DETAILS IN 4D}

\section{A. Overall smearing strategy}

Given the results in the previous three sections, the combination of "isotropic derivative" and "Brillouin Laplacian" seems most attractive. In other words, our preferred operator is

$$
\begin{aligned}
D(x, y)= & \sum_{\mu} \gamma_{\mu} \nabla_{\mu}^{\mathrm{iso}}(x, y)-\frac{a}{2} \triangle^{\mathrm{bri}}(x, y)+m_{0} \delta_{x, y} \\
& -\frac{c_{\mathrm{SW}}}{2} \sum_{\mu<\nu} \sigma_{\mu \nu} F_{\mu \nu} \delta_{x, y}
\end{aligned}
$$

and below we shall refer to it as the "Brillouin operator."

An ingredient which has proven particularly useful in the design of fermion actions with small cutoff effects is link smearing, also known under the label of "fat links" [25-30]. In the quenched QCD tests reported below a single step of APE smearing [31]

\footnotetext{
${ }^{4}$ Note the difference to naive or staggered massless fermions in $4 \mathrm{D}$, where both chiralities sit on top of each other. For the effect of nonstandard staggered mass terms and the resulting eigenvalue spectra, see $[23,24]$.
}

$$
\begin{aligned}
V_{\mu}(x) \equiv & U_{\mu}^{\mathrm{APE}}(x) \\
= & P_{S U(3)}\left\{(1-\alpha) I+\frac{\alpha}{6} \sum_{ \pm \nu \neq \mu} U_{\nu}(x) U_{\mu}(x+\hat{\nu})\right. \\
& \left.\times U_{\nu}^{\dagger}(x+\hat{\mu}) U_{\mu}^{\dagger}(x)\right\} U_{\mu}(x)
\end{aligned}
$$

with $\alpha=0.72$ is applied (for the details of the back projection to $S U(3)$, see Sub. V B). The result is used as an input in the covariant derivative and the covariant Laplacian. The latter operators are made gauge covariant in the simplest possible way, by summing over all shortest paths, with subsequent back projection to $S U(3)$. For instance the hyperdiagonal connections (4 hops) receive contributions from 24 paths, while the cubic-diagonal connections ( 3 hops) receive 6 contributions, and the square diagonals ( 2 hops) just 2.

We use the same kind of smeared gauge links $V_{\mu}(x)$ in the construction of the derivative, the Laplacian, and the fieldstrength tensor. Since this change is ultralocal and modifies only operators of mass dimension 5 and higher, the universality class of the action is unaffected. Other smearing strategies are possible, e.g., only relevant pieces or only irrelevant pieces of the action may be smeared. However, as we are unaware of any advantage of such more complicated schemes, we prefer to stay with the overall smearing strategy where all "thin" links $U_{\mu}(x)$ in (14), even if within $F_{\mu \nu}$, are replaced by the same kind of "fat" links $V_{\mu}(x)$.

The goal of our quenched scaling study in Sec. VI is to confront (14) with the standard Wilson action. To compare like with like, we will use the same smearing strategy and the same kind of clover improvement with $c_{\mathrm{SW}}=1$ (see Sub. V C below) in either case.

For completeness, let us mention that in order to simulate full QCD with a fat-link Brillouin or Wilson action and an hybrid Monte Carlo algorithm, one would equip either action with a smearing that is tailored to this purpose (e.g., "stout/ EXP" [32], "n-APE" [33], "LOG" [34], "over-improved stout" [35]).

\section{B. Details of the projection to $S U(N)$}

The projection of an arbitrary $N \times N$ matrix $A$ to $S U(N)$ is usually defined through a projection to $U(N)$, followed by a projection to unit determinant. The first projection is realized as

$$
P_{U(N)}\{A\}=A\left(A^{\dagger} A\right)^{-1 / 2}=\left(A A^{\dagger}\right)^{-1 / 2} A,
$$

where the equivalence of the two representations follows from the singular value decomposition $A=U S V^{\dagger}$, with unitary $U, V$, and $S>0$, resulting in $P_{U(N)}(A)=U V^{\dagger}$. Since $A^{\dagger} A$ and $A A^{\dagger}$ are both Hermitean, either version of (16) requires only one eigen system.

The projection to unit determinant is somewhat more involved, even if we restrict the discussion to unitary arguments, as suggested by the above 2 -step procedure. The 
most naive recipe is to divide a given $U \in U(N)$ by the $N$ th root of its determinant. Unfortunately, this is not a valid procedure, since there is a finite (nonzero) likelihood ${ }^{5}$ that the argument has $\operatorname{det}(U)=-1$, which lies on the branch cut. It is thus necessary, in general, to distribute the phase rotation (to go from det $=e^{\mathrm{i} \phi}$ to $\operatorname{det}=1$ ) unevenly among the $N$ eigenvalues.

In our opinion, a particularly compelling option for fixing this ambiguity is to notice that the $U(N)$ projection defined in (16) can be understood as the result of the recipe

$$
P_{U(N)}\{A\}=\min _{X \in U(N)} \operatorname{tr}\left\{(A-X)^{\dagger}(A-X)\right\}
$$

and to define the complete projection by using this recipe for the $S U(N)$ group, i.e., via

$$
P_{S U(N)}\{A\}=\min _{Y \in S U(N)} \operatorname{tr}\left\{(A-Y)^{\dagger}(A-Y)\right\}
$$

in a single step, where an algorithmic solution has been proposed in [36]:

(1) Perform a singular value decomposition $A=U S V^{\dagger}$ with $U, V \in U(N)$, and $S>0$ a diagonal matrix with positive entries. Indeed, $X=U V^{\dagger}$ is the projection to $U(N)$, but $\operatorname{det}(X) \neq 1$.

(2) Compute $\operatorname{det}(A)=\rho \exp (\mathrm{i} \phi)$. Incidentally, $\operatorname{det}(S)=\rho$ and $\operatorname{det}\left(U V^{\dagger}\right)=\exp (\mathrm{i} \phi)$. The matrix $U \exp (-\mathrm{i} \phi / N) V^{\dagger}$ is in $S U(N)$, but it is, in general, not the one which is closest to $A$.

(3) Find the solution $\left\{\theta_{i}\right\}$ for the phases of the matrix $D=\operatorname{diag}\left(\exp \left(\mathrm{i} \theta_{1}\right), \ldots, \exp \left(\mathrm{i} \theta_{N}\right)\right)$, subject to the constraint $\sum \theta_{i}+\phi=0(\bmod 2 \pi)$, which maximizes $\operatorname{Re} \operatorname{Tr}\left(A^{\dagger} U D V^{\dagger}\right)$. By means of the original singular value decomposition, the latter expression equals $\operatorname{Re} \operatorname{Tr}(S D)$, and the expression to be maximized $^{6}$ is $s_{1} \cos \left(\theta_{1}\right)+\ldots+s_{N} \cos \left(\theta_{N}\right)$, still subject to the constraint $\sum \theta_{i}+\phi=0(\bmod 2 \pi)$. The matrix $Y=U D V^{\dagger}$ is the desired solution.

From (18), it is clear that, if $A$ is subject to a random gauge transformation $A \rightarrow g_{1} A g_{2}^{\dagger}$ with $g_{1,2} \in S U(N)$, the effect must be that the solution $Y$ transforms as

${ }^{5}$ The issue arises if $A$ is the sum of only two $S U(N)$ matrices. The determinant of $A=U+V$ is real, since

$$
\begin{aligned}
\operatorname{det}(U+V) & =\operatorname{det}\left(U\left(V^{\dagger}+U^{\dagger}\right) V\right)=\operatorname{det}\left(V^{\dagger}+U^{\dagger}\right) \\
& =[\operatorname{det}(U+V)]^{*}
\end{aligned}
$$

with no generalization if $A$ is a sum of three and more unitary matrices. Hence, with arbitrary $U, V \in S U(N)$ it may happen that $\operatorname{det}(U+V)$ lies on the negative real axis. Taking a look at (16), one realizes that $\operatorname{det}(A)<0$ implies $\operatorname{det}(B)=-1$, where $B=P_{U(N)}\{A\}$ and $A$, the original sum of two $S U(N)$ matrices.

${ }^{6}$ Note that the global maximum is required. A possible strategy is to perform a scan, in regularly spaced intervals, over all unconstrained $\theta_{i}$, followed by a local maximization starting from the largest value obtained in the survey. Thus, staying content with intervals $\Delta \theta_{i}=\pi / 3$, the global scan requires $6^{N-1}$ function calls.
$Y \rightarrow g_{1} Y g_{2}^{\dagger}$. Up to a set of gauge configurations of measure zero, the singular value decomposition $A=U S V^{\dagger}$ is unique (here we assume a specific ordering in $S$, e.g., $\left.s_{1}>\ldots>s_{N}>0\right)$. As a result, the effect of the random gauge transformation is just $U \rightarrow g_{1} U, V \rightarrow g_{2} V$, while $S$ and the expression to be maximized are unchanged, and the net effect is thus $U D V^{\dagger} \rightarrow g_{1} U D V^{\dagger} g_{2}^{\dagger}$, as expected.

\section{Tree-level improvement}

The Symanzik effective field theory of cutoff effects of undoubled lattice Dirac operators is based on an analysis of all local mass dimension 5 operators consistent with the symmetries of the theory [5-9]. As long as one is content with perturbative or nonperturbative $O(a)$-improvement of on shell Green's functions, contact terms can be ignored and it suffices to add a single improvement term, the socalled clover term which is included in (1) and (14). Going through the arguments of [5-9], one realizes that the leading contribution, in the weak coupling expansion, to the coefficient in front is independent of the details of the covariant derivative and Laplacian occurring in the operator. In other words, $c_{\mathrm{SW}}=1$ holds true, at tree level, also for our Brillouin operator (14), while subleading contributions are, of course, different.

With $c_{\mathrm{SW}}=1$, the leading cutoff effects are $O(\alpha a)$, and the overall smearing strategy does not change this. However, due to the smearing, the coefficient of the $O(\alpha a)$ term might be so small that the formally subleading $O\left(a^{2}\right)$ cutoff effects might prove numerically dominant.

\section{PRACTICAL TESTS IN QUENCHED QCD}

\section{A. Scale setting and overall tuning strategy}

We use the Wilson gauge action and a parametrization of $r_{0} / a$ consistent with asymptotics [37]

$$
\log \left(r_{0} / a\right)=\frac{4 \pi^{2}}{33} \beta \frac{1-8.2384 / \beta+15.310 / \beta^{2}}{1-2.7395 / \beta-11.526 / \beta^{2}},
$$

which is based on data from [38]. Upon choosing $L / a=10,12,16,20,24$ and requesting that $L / r_{0}=$ 3.2653 (tantamount to $L \simeq 1.6 \mathrm{fm}$ if $r_{0} \simeq 0.49 \mathrm{fm}$ is assumed), we find that we should use the $\beta$ values listed in Table I. Note that the quenched scale ambiguity of $\sim 5 \%$ does not limit our ability to match boxes in terms of $L / r_{0}$.

We aim for comparisons at a fixed value of the light and the strange quark mass. This will be achieved by tuning the pion and the $\bar{s} s$ mass (without disconnected contributions) to $\left(r_{0} M_{\pi}\right)^{2} \simeq 1.56 \simeq 1.25^{2}$ and $\left(r_{0} M_{\bar{s} s}\right)^{2} \simeq 4.56$, respectively. Given that $M_{\bar{s} s}^{2} \simeq 2 M_{K}^{2}-M_{\pi}^{2}$, this will correspond to $\left(r_{0} M_{K}\right)^{2} \simeq 3.06 \simeq 1.75^{2}$, and hence to a pion mass of about $500 \mathrm{MeV}$ and a kaon mass of about $700 \mathrm{MeV}$. Finite volume effects are expected to be small, since $M_{\pi} L \simeq 4.08$. 
TABLE I. Summary of $\kappa_{\text {crit }}$ for either operator with 1 APE step and $c_{\mathrm{SW}}=1$, as determined from direct measurements at these couplings (with error bars) and through the fit (21).

\begin{tabular}{|c|c|c|c|c|c|c|}
\hline \multirow{2}{*}{$\frac{\beta}{5.72}$} & \multirow{2}{*}{$\frac{\text { Geom. }}{10^{3} \times 20}$} & \multirow{2}{*}{$\frac{a^{-1}[\mathrm{GeV}]}{1.236}$} & \multicolumn{2}{|c|}{$\kappa_{\text {crit }}^{\text {std } / \text { std }}$ (standard) } & \multicolumn{2}{|c|}{$\kappa_{\text {crit }}^{\text {iso/bri }}$ (Brillouin) } \\
\hline & & & $0.134516(65)$ & 0.134533 & $0.129780(64)$ & 0.129798 \\
\hline 5.80 & $12^{3} \times 24$ & 1.479 & $0.132673(47)$ & 0.132650 & $0.128594(30)$ & 0.128582 \\
\hline 5.95 & $16^{3} \times 32$ & 1.978 & $0.130760(59)$ & 0.130769 & $0.127469(48)$ & 0.127471 \\
\hline 6.08 & $20^{3} \times 40$ & 2.463 & $0.129818(45)$ & 0.129864 & $0.126940(30)$ & 0.126973 \\
\hline 6.20 & $24^{3} \times 48$ & 2.964 & $0.129362(57)$ & 0.129303 & $0.126725(42)$ & 0.126676 \\
\hline
\end{tabular}

\section{B. Determination of $\boldsymbol{\kappa}_{\text {crit }}$ and choice of $\kappa_{\text {light }}, \kappa_{\text {strange }}, \kappa_{\text {charm }}$}

As a first step, we determine $\kappa_{\text {crit }}$ for either operator (with $1 \mathrm{APE}$ step and $c_{\mathrm{SW}}=1$ ) over a range of $\beta$-values, with results given in Table I and Fig. 10. The perturbative formula reads $\left(\beta=6 / g_{0}^{2}\right)$

$$
a m_{\text {crit }}=\Sigma_{0}=-\frac{g_{0}^{2}}{16 \pi^{2}} C_{F} S+O\left(g_{0}^{4}\right) \quad[<0]
$$

with $C_{F}=4 / 3$ and $a m_{\text {crit }}=1 /\left(2 \kappa_{\text {crit }}\right)-4$. At 1-loop order, one finds $S=31.98644$ for the thin-link Wilson operator with $c_{\mathrm{SW}}=1$, and $S=4.07175$ for the $1 \mathrm{APE}$ $(\alpha=0.72)$ variety with $c_{\mathrm{SW}}=1$ [30], while for the Brillouin operator no perturbative information is available.

To see how far from the perturbative regime we are, we fit our data to the rational ansatz

$$
-a m_{\text {crit }}=\frac{c_{1} g_{0}^{2}+c_{2} g_{0}^{4}}{1+c_{3} g_{0}^{2}}
$$

with 2 degrees of freedom, and compare the fitted $c_{1}$ to the 1-loop prediction $S /\left(12 \pi^{2}\right)$, where available. For the

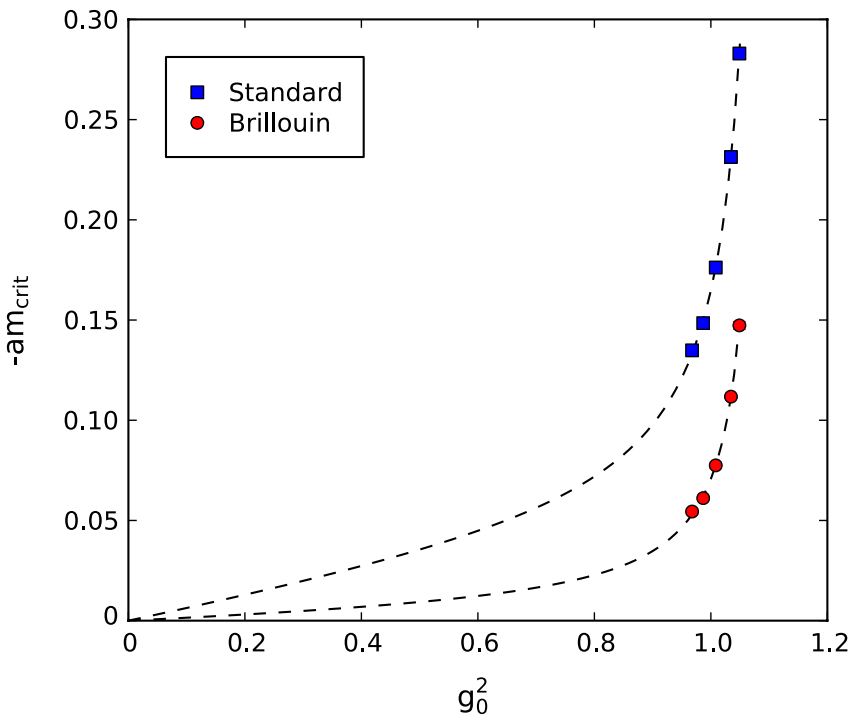

FIG. 10 (color online). Additive mass renormalization versus $g_{0}^{2}$ for the Wilson operator and the Brillouin operator (both with $c_{\mathrm{SW}}=1$ and $\alpha_{\mathrm{APE}}=0.72$ ). In either case a rational fit with the ansatz (21) is included. Error bars are significantly smaller than the size of the symbols.
Wilson operator, $c_{1}=0.0623(82)$ deviates significantly from the prediction 0.0344, while for the Brillouin operator $c_{1}=0.0143(54)$, without a perturbative prediction to compare to. Given the quality of these fits, the interpolated $\kappa_{\text {crit }}$ are more accurate than the direct measurements, and this is why we include these values in Table I.

In order to perform a quenched scaling study, we define, for each $\beta$, three reference $\kappa$-values which realize $\left(r_{0} M_{\pi}\right)^{2}=1.56,\left(r_{0} M_{\bar{s} s}\right)^{2}=4.56$, and $\left(r_{0} M_{\bar{c} c}\right)^{2}=46.5$. We call them $\kappa_{\text {light }}, \kappa_{\text {strange }}$, and $\kappa_{\text {charm }}$, respectively, (even though the first two are heavier than the respective physical flavors, and the last one is lighter than the physical charm quark). These values are determined, for each coupling, by interpolating the results of a few tuning runs. The three reference $\kappa$-values are then evaluated on the full ensembles, and the resulting $\left(r_{0} M_{P}\right)^{2}$ are compared to the target values in Fig. 11. It seems the tuning is accurate enough, so that we can proceed with a study of the scaling of the decay constants.

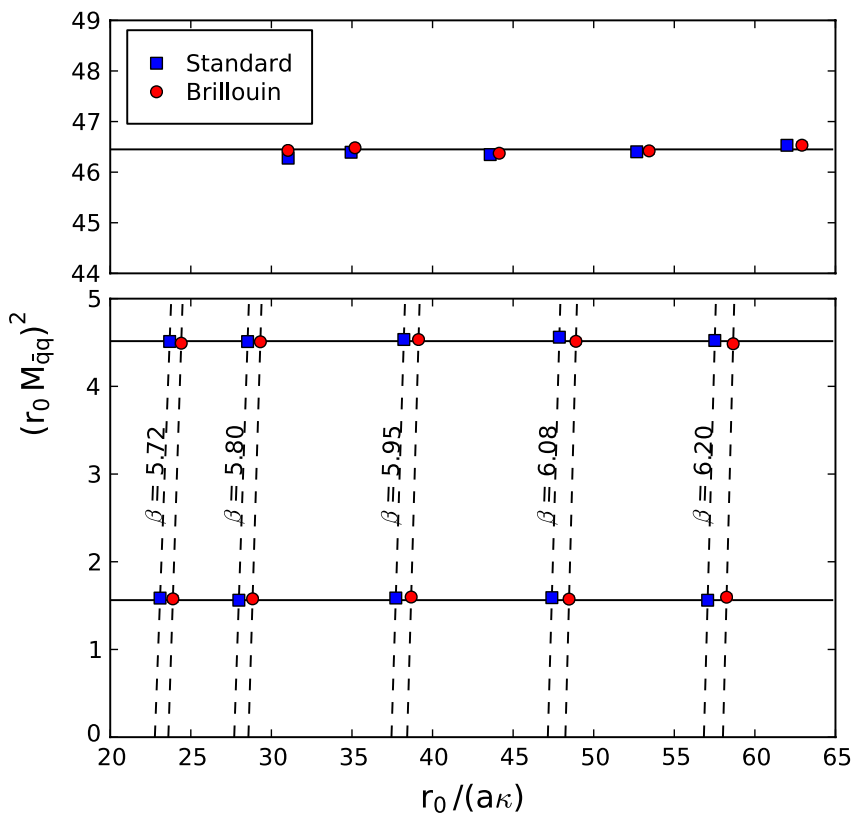

FIG. 11 (color online). Summary of the final $\left(r_{0} M_{\pi}\right)^{2}$, $\left(r_{0} M_{\bar{s} s}\right)^{2},\left(r_{0} M_{\bar{c} c}\right)^{2}$ to test how accurately the target values $1.56,4.56,46.5$ were reached. Error bars are smaller than the size of the symbols. 


\section{Comparing the scaling of decay constants at fixed $r_{0} M_{P}$}

The decay constants $F_{\pi}, F_{\bar{s} s}, F_{\bar{c} c}$ are determined from the improved and renormalized current
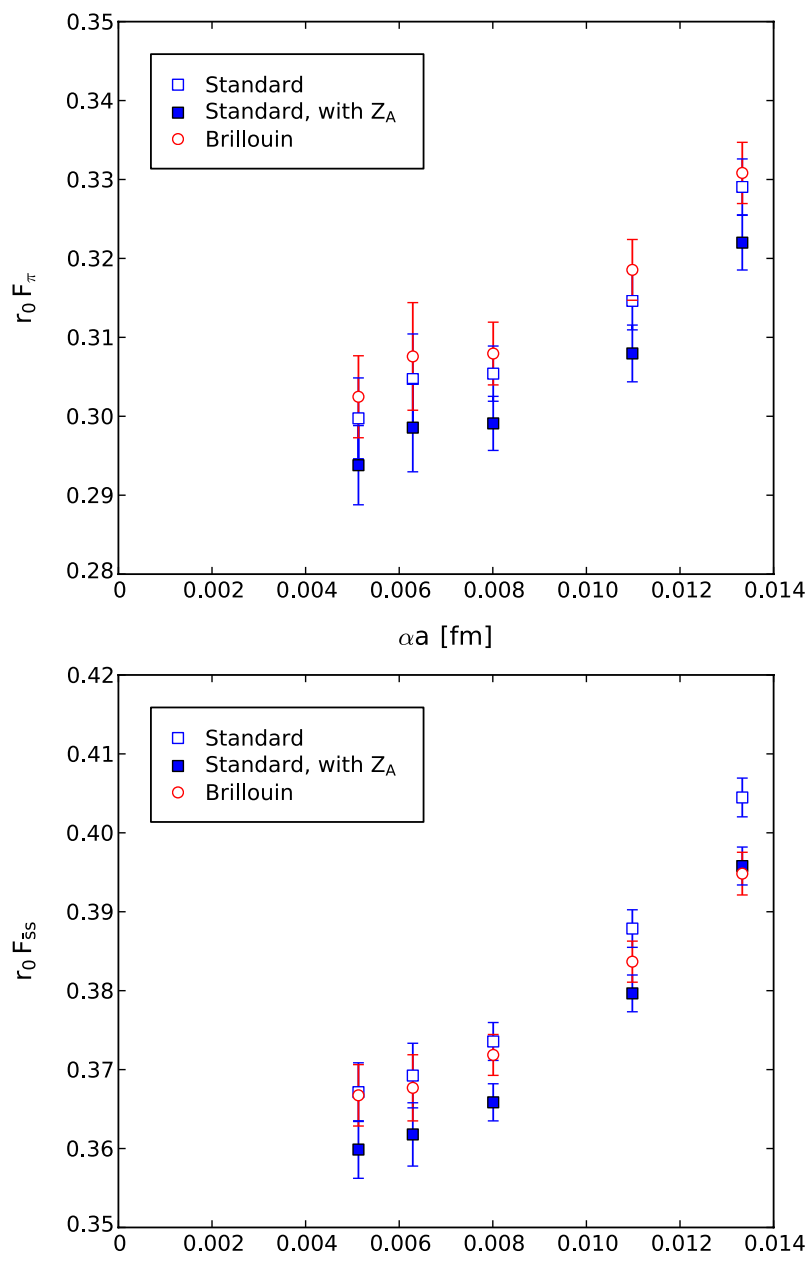

$\alpha \mathrm{a}[\mathrm{fm}]$

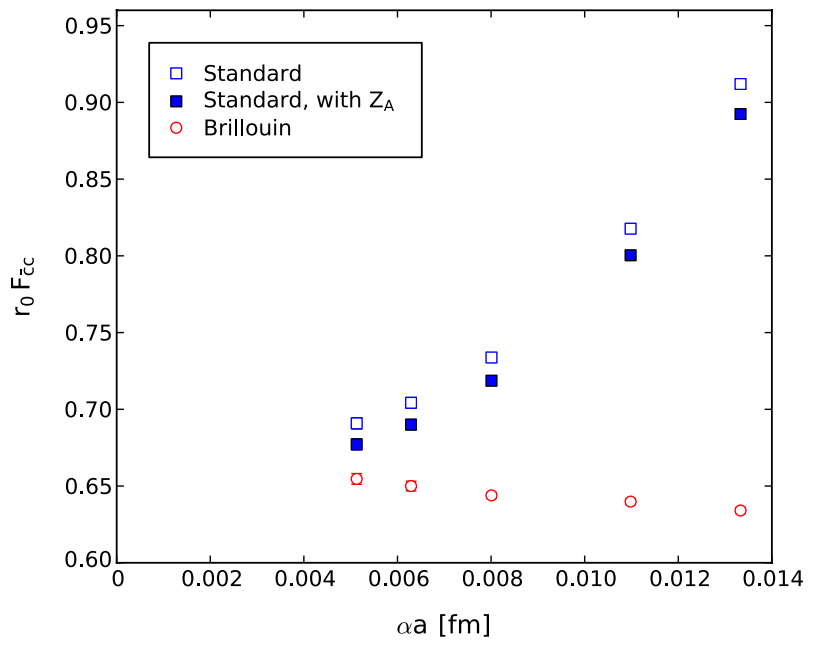

$$
A_{\mu}^{\mathrm{ren}}=Z_{A}\left(1+b_{A} a m^{\mathrm{W}}\right)\left(A_{\mu}+a c_{A} \bar{\partial}_{\mu} P\right),
$$

where $A_{\mu}$ and $P$ denote the naive axial-vector current and pseudoscalar density, respectively, and $m^{\mathrm{W}}=m_{0}-m_{\text {crit }}$.
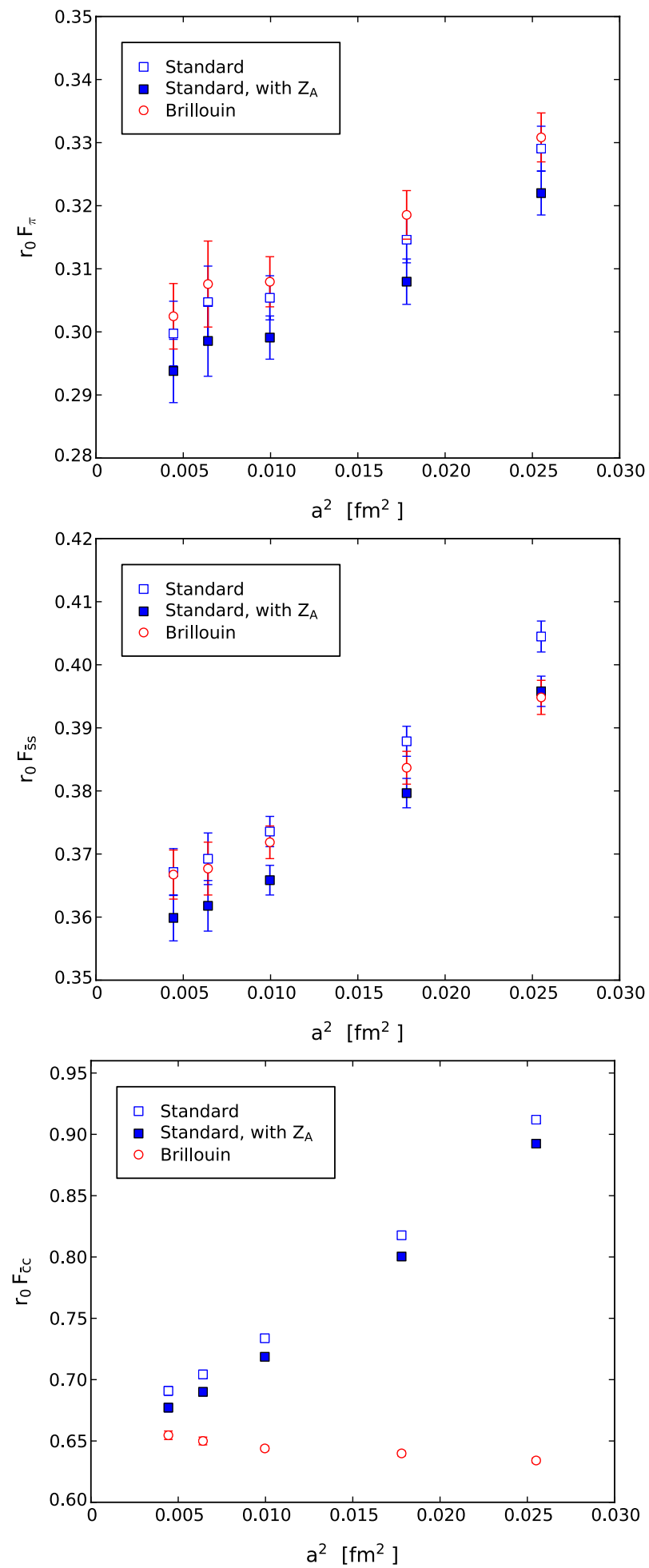

FIG. 12 (color online). Decay constants $F_{\pi}$ (top), $F_{\bar{s} s}$ (middle), $F_{\bar{c} c}$ (bottom) in $r_{0}$ units versus $\alpha a$ (left) and $a^{2}$ (right). Open symbols indicate the bare values and filled symbols include the 1-loop $Z_{A}$. 
In practice, $m^{\mathrm{W}}$ in (22) is often replaced by the PCAC quark mass

$$
m^{\mathrm{PCAC}}=\frac{\sum_{\mathbf{x}}\left\langle\bar{\partial}_{4}\left[A_{4}(x)+a c_{A} \bar{\partial}_{4} P(x)\right] O(0)\right\rangle}{2 \sum_{\mathbf{x}}\langle P(x) O(0)\rangle}
$$

where $\bar{\partial}$ denotes the symmetric derivative, and usually $O \equiv$ $P$ is chosen to get maximal signal. Here it is assumed that the two quark masses are equal; in general the improvement factor in (22) is $\left(1+b_{A} a\left(m_{j}^{\mathrm{W}}+m_{k}^{\mathrm{W}}\right) / 2\right)$ for flavors $j, k$, and the left-hand side of (23) is $\left(m_{j}^{\mathrm{PCAC}}+m_{k}^{\mathrm{PCAC}}\right) / 2$.

We use the tree-level improvement coefficients $b_{A}=1$, $c_{A}=0$. The 1-loop renormalization constant $Z_{A}=$ $1-g_{0}^{2} z_{A} /\left(12 \pi^{2}\right)$, which is needed for consistency, is known for the Wilson operator $\left(z_{A}=2.42423\right.$ with 1 step of $\alpha_{\mathrm{APE}}=0.72$ smearing and $c_{\mathrm{SW}}=1$ is found in [30]), but not for the Brillouin operator. In Fig. 12, we plot the decay constants $F_{\pi}, F_{\bar{s} s}, F_{\bar{c} c}$ versus $\alpha a$ (left) and $a^{2}$
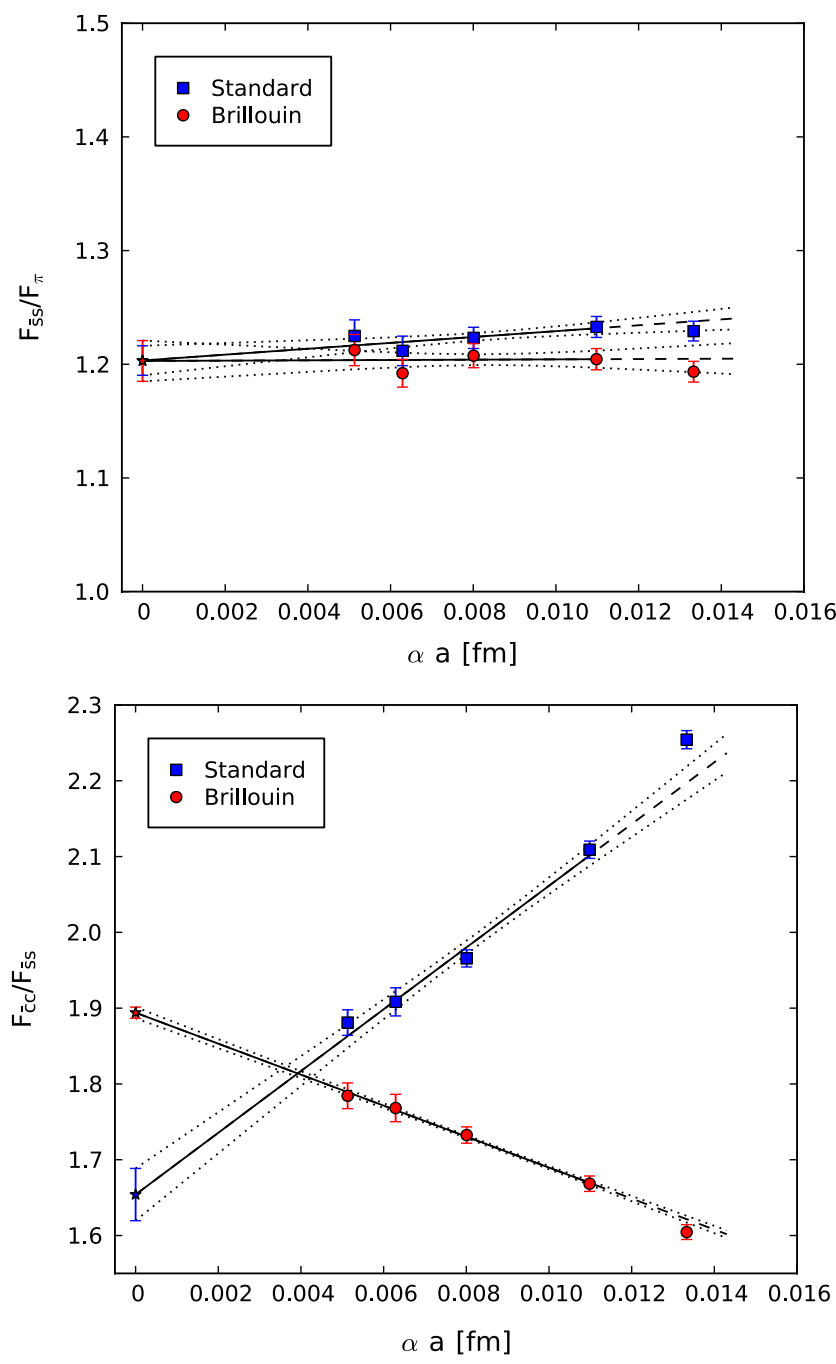

(right). Here everything is made dimensionless through $r_{0}$. In the case of the Wilson operator, the lattice-to-continuum matching factor $Z_{A}$ is included, but it brings a rather small shift, since it is already close to 1 in the range of couplings where we have data, and it approaches 1 as $a \rightarrow 0$. With hindsight, we can thus anticipate that also for the Brillouin operator the data without $Z_{A}$ are indicative of the approach to the continuum. Comparing the two operators without $Z_{A}$, we see little difference in the light and strange pseudoscalar data (top and middle), while there is a pronounced difference in the charm sector (bottom row). Hence, for the scaling of $r_{0} F_{\bar{c} c}$ the Brillouin operator seems to bring a significant improvement.

To get rid of the $Z_{A}$ factors, we also consider the scaling of the ratios $F_{\bar{s} s} / F_{\pi}$ and $F_{\bar{c} c} / F_{\bar{s} s}$, as shown in Fig. 13. Again, we plot the data against $\alpha a$ (left) and $a^{2}$ (right). For the strange-to-light ratio, all data happen to be essentially flat, so there is no advantage of one operator over the other. For the charm-to-strange ratio, the situation is different.
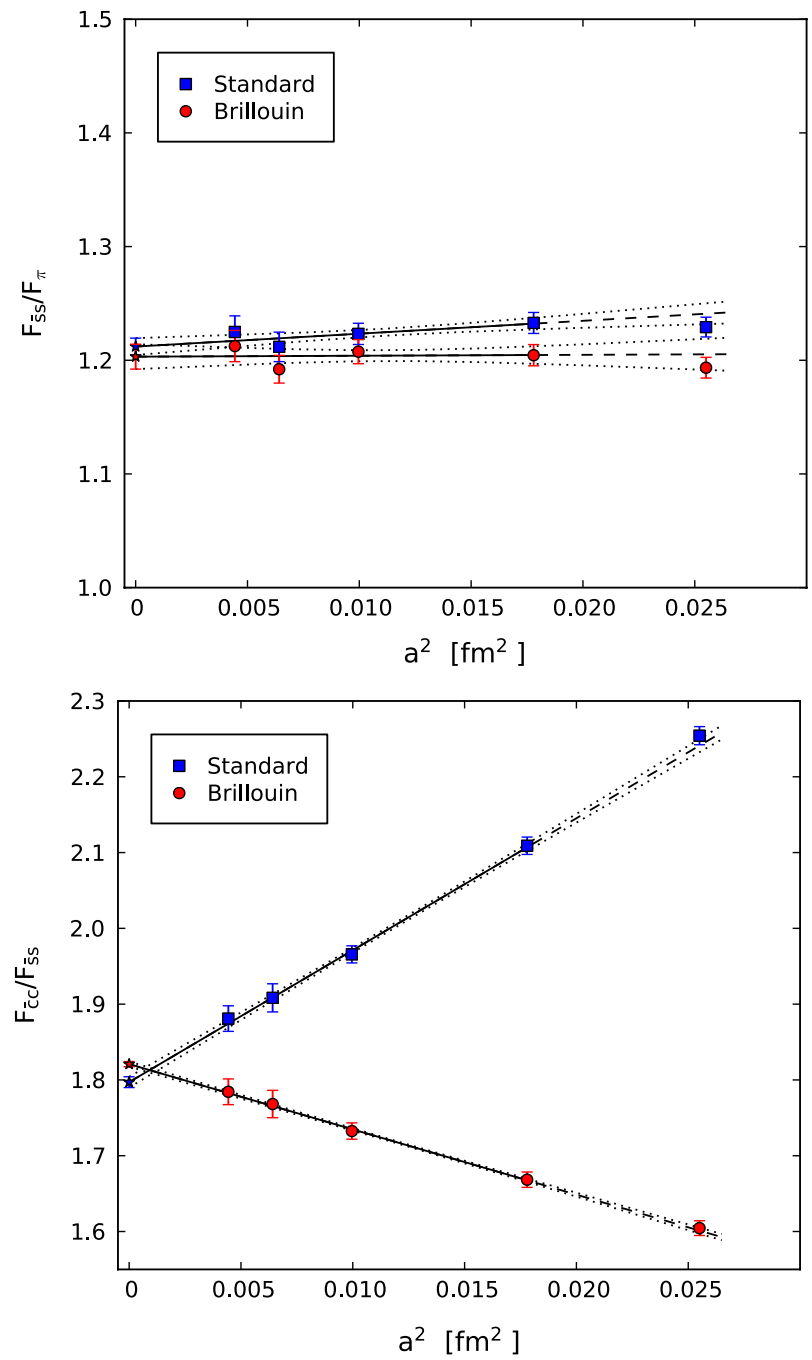

FIG. 13 (color online). The ratios $F_{\bar{s} s} / F_{\pi}$ (top) and $F_{\bar{c} c} / F_{\bar{s} s}$ (bottom) versus $\alpha a$ (left) and $a^{2}$ (right). The linear fits include only 4 lattice spacings and favor the pure $a^{2}$ over the pure $\alpha a$ extrapolation. 

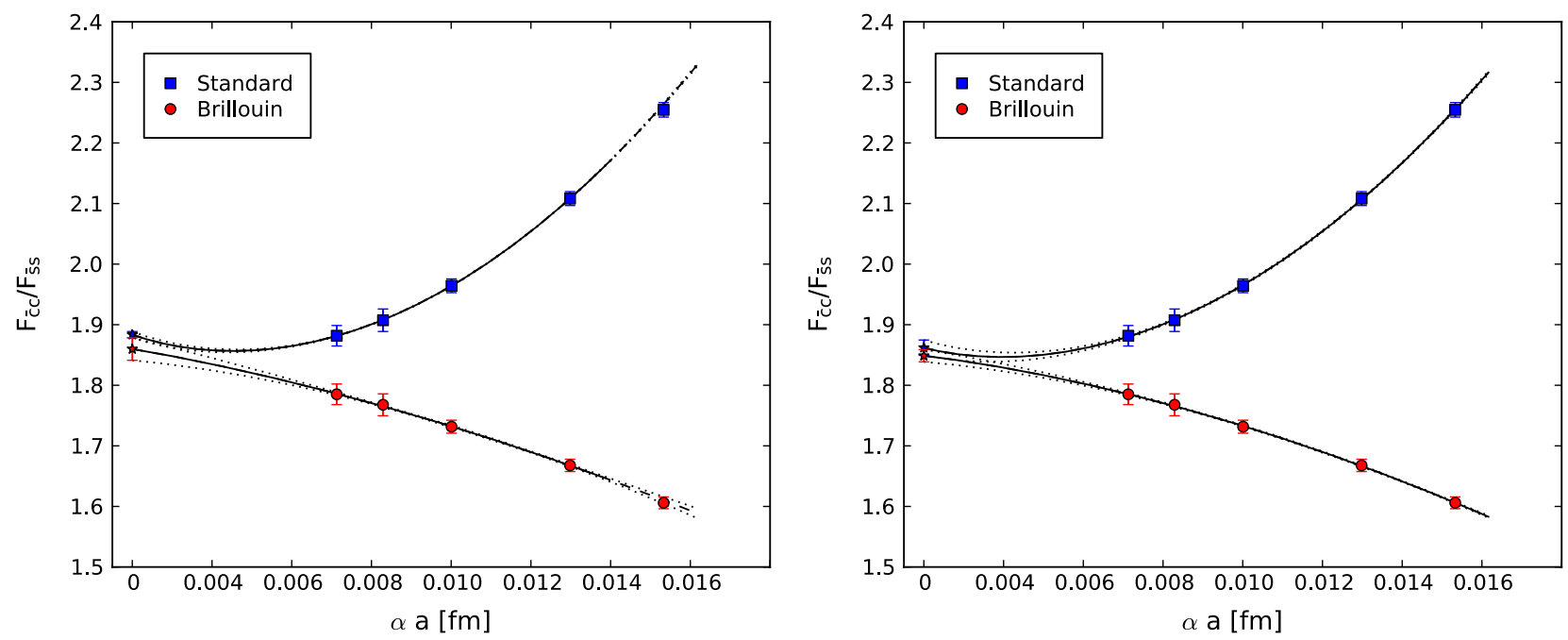

FIG. 14 (color online). Fit of the mixed $\alpha a$ plus $a^{2}$ ansatz (24) to the ratio $F_{\bar{c} c} / F_{\bar{s} s}$ with 4 (left) or 5 (right) lattice spacings included.

Fitting the data on the four finer lattices with a pure $\alpha a$ ansatz yields two continuum extrapolated results, which are not consistent (lower left panel). Fitting the same data with a pure $a^{2}$ ansatz leads to two continuum extrapolated results, which are almost consistent (lower right panel). If we restrict the fits to the three finest lattice spacings, the values obtained with the pure $\alpha a$ hypothesis stay inconsistent, while the continuum results with the pure $a^{2}$ hypothesis become consistent. To prevent any misunderstanding, let us emphasize that we think that both operators have a contribution in $\alpha a$ and $a^{2}$ at accessible lattice spacings. Still, to the best of our knowledge, this is the first figure which indicates that, for a tree-level improved operator with some link smearing, the pure $a^{2}$ hypothesis might be closer to the truth than the (formally correct) pure $\alpha a$ hypothesis. Of course, with infinitely precise data, one could separate the two contributions. To see how far we are

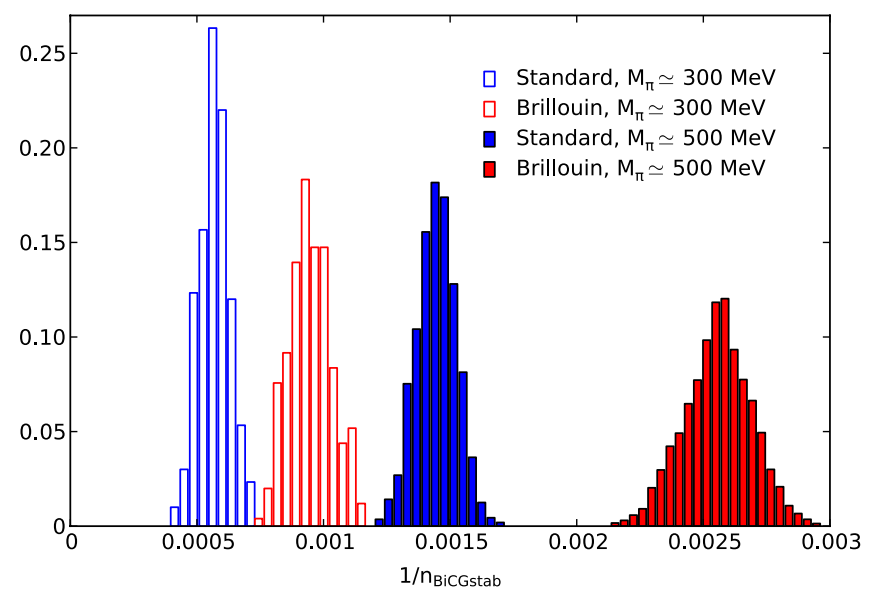

FIG. 15 (color online). Distribution of the inverse iteration count $1 / n_{\text {BiCGstab }}$ to reach a norm $\epsilon=10^{-7}$ of the residual at $\beta=6.20$ with $\kappa$ tuned to have $\left(r_{0} M_{\pi}\right)^{2}=1.56$ ("500 MeV") or 0.56 ("300 MeV"). from this ideal world, we try a fit of the ratio $F_{\bar{c} c} / F_{\bar{s} s}$ with the ansatz

$$
F_{\bar{c} c} / F_{\bar{s} s}=d_{0}+d_{1} \alpha(a) a+d_{2} a^{2}
$$

giving the results shown in Fig. 14. The fitted $d_{1}, d_{2}$ of the Brillouin operator are significantly smaller than those of the Wilson operator. Also by looking at the fits one would say that the Brillouin data alone leave little doubt that the correct continuum value is somewhere near 1.85 , while with the Wilson data alone this is far from obvious.

\section{Comparing the $1 / n_{\text {BiCGstab }}$ distributions at fixed $r_{0} M_{\pi}$}

In quenched QCD with Wilson fermions, so-called exceptional configurations (on which the massive Dirac operator $D_{m}$ could not be inverted) hindered the approach to light quark masses. In full QCD, the functional measure suppresses configurations on which $D_{m}$ has near-zero modes. Still, the issue persists in the form of instabilities in the hybrid Monte Carlo evolution.
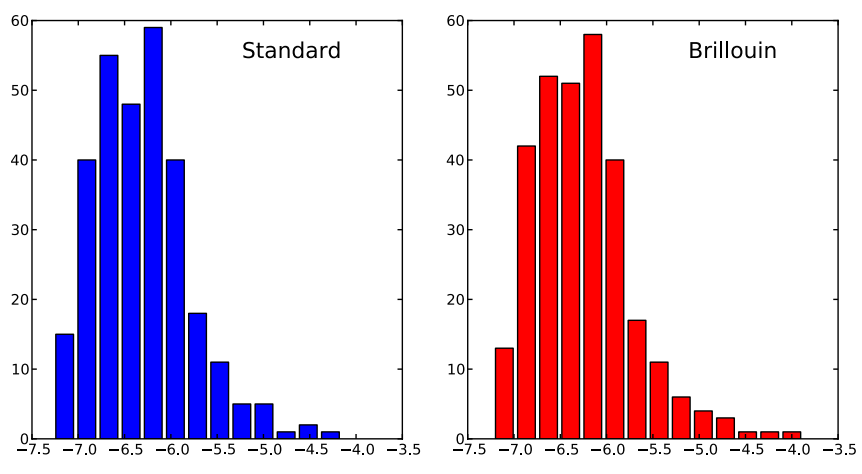

$\log [\operatorname{Corr}(T / 4)]$

FIG. 16 (color online). Distribution of the logarithm of the correlator $P(T / 4) \bar{P}(0)$ at $\beta=6.20$ with $\kappa$ tuned to have $\left(r_{0} M_{\pi}\right)^{2}=1.56$ in either case. 

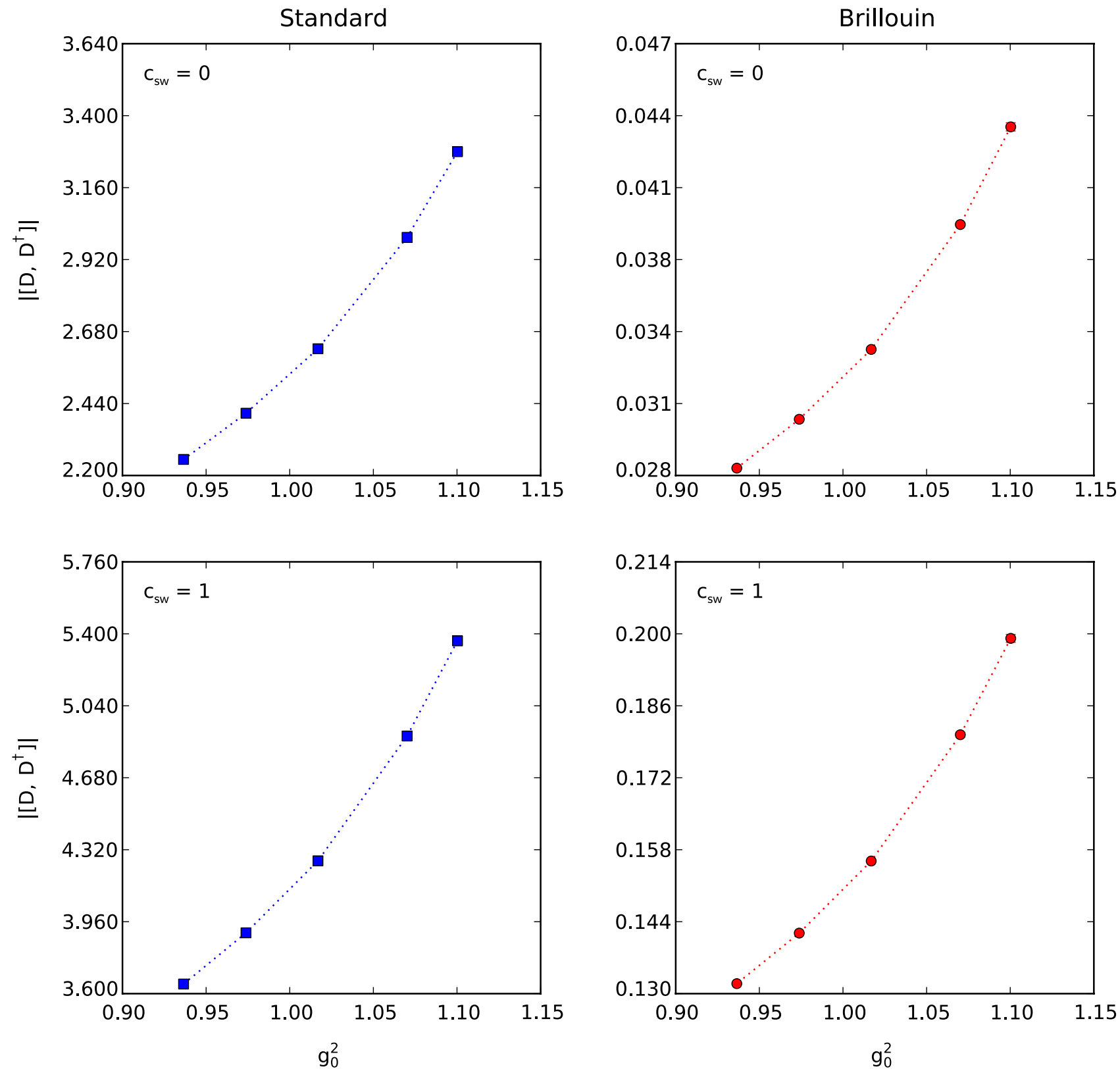

FIG. 17 (color online). Non-normality $\left\|\left[D, D^{\dagger}\right]\right\|$ versus $g_{0}^{2}$, after 1 APE step, for the Wilson (left) and Brillouin (right) operator, with $c_{\mathrm{SW}}=0$ (top) and 1 (bottom). Note the difference in scale.

In [39], it was shown that the stability of these simulations is linked to the distribution of the lowest eigenvalue of $D_{m}^{\dagger} D_{m}$. The latter is roughly Gaussian distributed, and the simulation is deemed safe as long as the center of the distribution is at least 4 standard deviations away from zero. The BMW Collaboration noticed that the smallest eigenvalue of $D_{m}^{\dagger} D_{m}$ is directly related to the number of iterations in the inversion and used the inverse iteration count $1 / n_{\mathrm{CG}}$ in the monitoring [40]. In Fig. 15, we present $1 / n_{\text {BiCGstab }}$ for either operator at the values $\left(r_{0} M_{\pi}\right)^{2}=$ 1.56 and $0.56\left(M_{\pi} \sim 500\right.$ and $\left.300 \mathrm{MeV}\right)$. In either case, an inversion with the Brillouin operator requires about
$60 \%$ of the forward applications ${ }^{7}$ of the Wilson operator. Finally, according to the safety criterion mentioned above, there seems to be a slight advantage for the Brillouin operator at low $M_{\pi}$. Using another $\beta$-value did not bring any major change.

\footnotetext{
${ }^{7}$ For fixed $M_{\pi}$, the smallest eigenvalues of the two $A=D_{m}^{\dagger} D_{m}$ are approximately equal, while the largest eigenvalue is near $2.5^{2}$ for the Brillouin operator and near $7.5^{2}$ for the Wilson operator. Since $n_{\mathrm{CG}} \propto \sqrt{\mathrm{CN}(A)}$ one would expect the relative iteration count to be around $1 / 3$ for $C G$ and around $1 / \sqrt{3} \simeq 0.6$ for BiCGstab.
} 


\section{E. Comparing the statistical fluctuations at fixed $\boldsymbol{r}_{\mathbf{0}} \boldsymbol{M}_{\pi}$}

Reaching the same statistics for Wilson and Brillouin data may or may not be a good guide to obtain equally precise physics results. To compare the fluctuations with either operator, we compare the variance in the correlator $C_{P \bar{P}}(t)$ at $t=T / 4$. The result is shown in Fig. 16 in the form of a histogram, with either $\kappa$ tuned to realize $\left(r_{0} M_{\pi}\right)^{2}=1.56$. Essentially, there is no noticeable difference between the two operators. Looking at other $\beta$-values we arrived at the same conclusion.

\section{SUITABILITY AS OVERLAP KERNEL}

\section{A. Details of the overlap action}

Given any undoubled (flavor symmetry respecting) "kernel" Dirac operator $D_{\mathrm{kn}, m}$ at a quark mass $m$, the massless overlap operator is defined through [3]

$$
\begin{aligned}
D_{\mathrm{ov}} & =D_{\mathrm{ov}, 0}=\frac{\rho}{a}\left[1+D_{\mathrm{kn},-\rho / a}\left(D_{\mathrm{kn},-\rho / a}^{\dagger} D_{\mathrm{kn},-\rho / a}\right)^{-1 / 2}\right] \\
& =\frac{\rho}{a}\left[1+\gamma_{5} \operatorname{sign}\left(\gamma_{5} D_{\mathrm{kn},-\rho / a}\right)\right],
\end{aligned}
$$

with $0<\rho<2$. Traditionally, the kernel parameter $\rho$ was tuned to a value above 1 to maximize the locality of $D_{\text {ov }}$ on coarse lattices [41]. However, on fine lattices (and with some link smearing or filtering of the kernel also on relatively coarse ones) maximal locality is obtained for $\rho<1[42,43]$. As $\rho$ is part of the action definition, it is desirable to keep it fixed, and we stay with the canonical choice $\rho=1$.

The massive overlap operator follows by adding a "chirally rotated" scalar term [44]

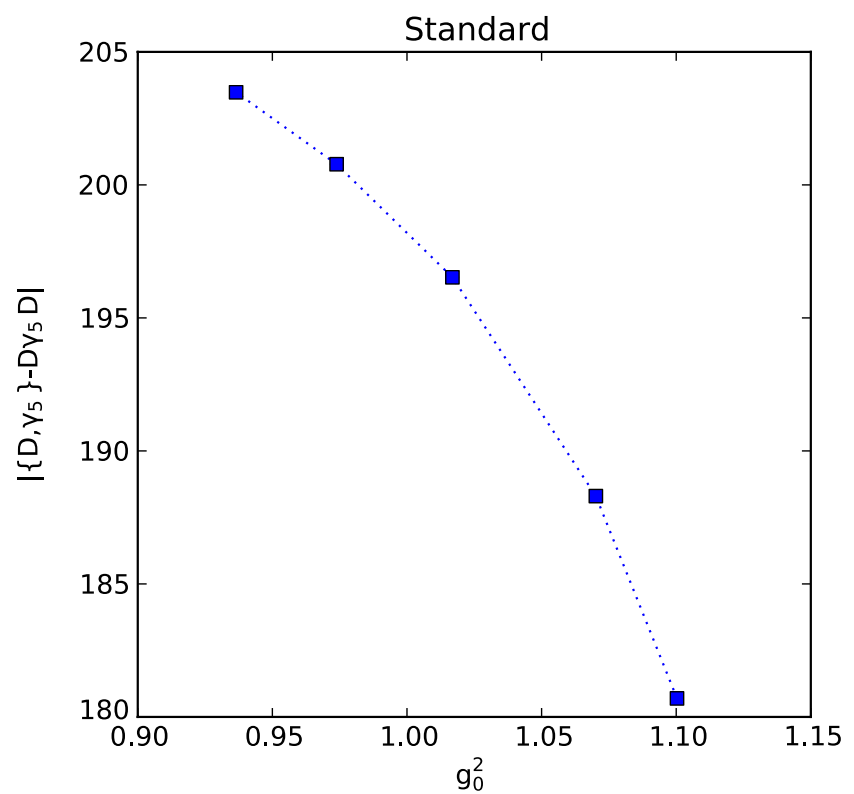

$D_{\mathrm{ov}, m}=D_{\mathrm{ov}}+m\left(1-\frac{a}{2 \rho} D_{\mathrm{ov}}\right)=\left(1-\frac{a m}{2 \rho}\right) D_{\mathrm{ov}}+m$,

which yields an operator with a circular eigenvalue spectrum of radius $\rho-a m / 2$ around the point $(\rho+a m / 2,0)$ in the complex plane.

There is still a choice to be made regarding the filtering of the underlying kernel operator (we use 1 and 5 APE steps) and whether one wants to equip it with a clover term.

\section{B. Comparing the near-normality of the kernels}

Wilson-type operators are usually non-normal, i.e., $\left[D, D^{\dagger}\right] \neq 0[45]$. This means that the spectral representation takes the form $D=\sum \lambda_{n}\left|\phi_{n}\right\rangle\left\langle\psi_{n}\right|$, with no simple connection between $\left|\phi_{n}\right\rangle$ and $\left\langle\psi_{n}\right|$ and, as a result of this, no simple connection between the eigenvalue spectra of $D$ and $D^{\dagger} D$. Chiral operators are usually normal, i.e., $\left[D, D^{\dagger}\right]=0$ for a staggered or overlap Dirac operator. This means that $D=\sum \lambda_{n}\left|\psi_{n}\right\rangle\left\langle\psi_{n}\right|$, with $\left|\psi_{n}\right\rangle$ and $\left\langle\psi_{n}\right|$ being the complex conjugate transpose of each other, and the spectrum of $D^{\dagger} D$ can be inferred from the one of $D$.

In this sense, one may understand the non-normality of a Wilson-type fermion, defined as the norm of the commutator, as a measure of "how far" it still is from a formulation with continuum-like features. Therefore, we measure $\left\|\left(D^{\dagger} D-D D^{\dagger}\right) \eta\right\|$ for a few dozen normalized Gaussian random vectors $\eta$, with $D$ being the Wilson or the Brillouin operator. By doing this on 15 configurations for each ensemble, we obtain the data shown in Fig. 17. Not surprisingly, with either operator the non-normality decreases towards the continuum, but the Brillouin operator fares significantly better. Surprisingly, switching on the clover term increases the non-normality, but it remains

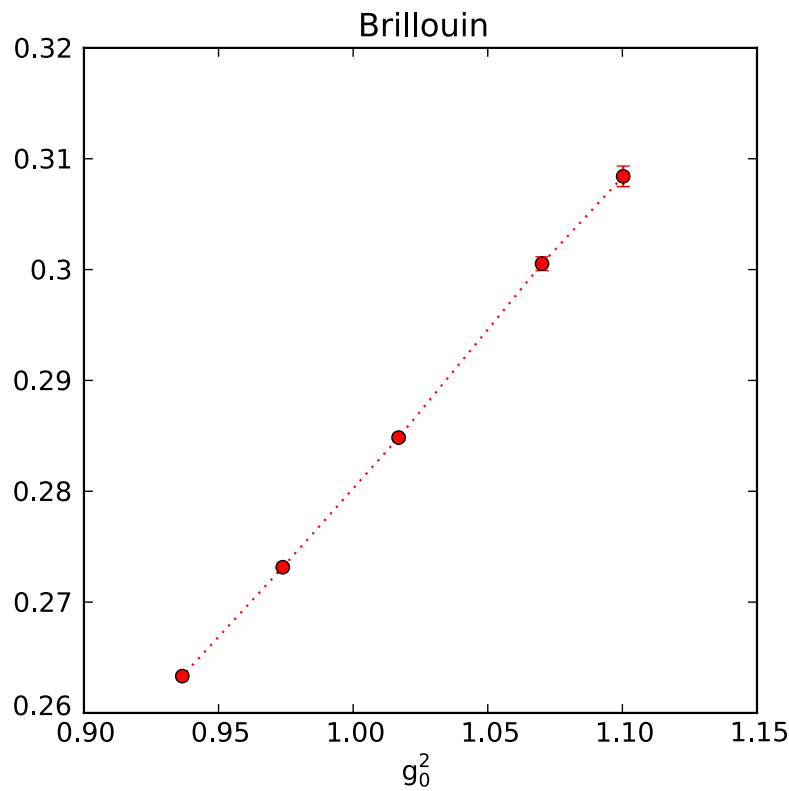

FIG. 18 (color online). Violation of the Ginsparg-Wilson relation (27) with $\rho=1$ as a function of $6 / \beta$ for either operator (see text for details). Note the difference in scale. 

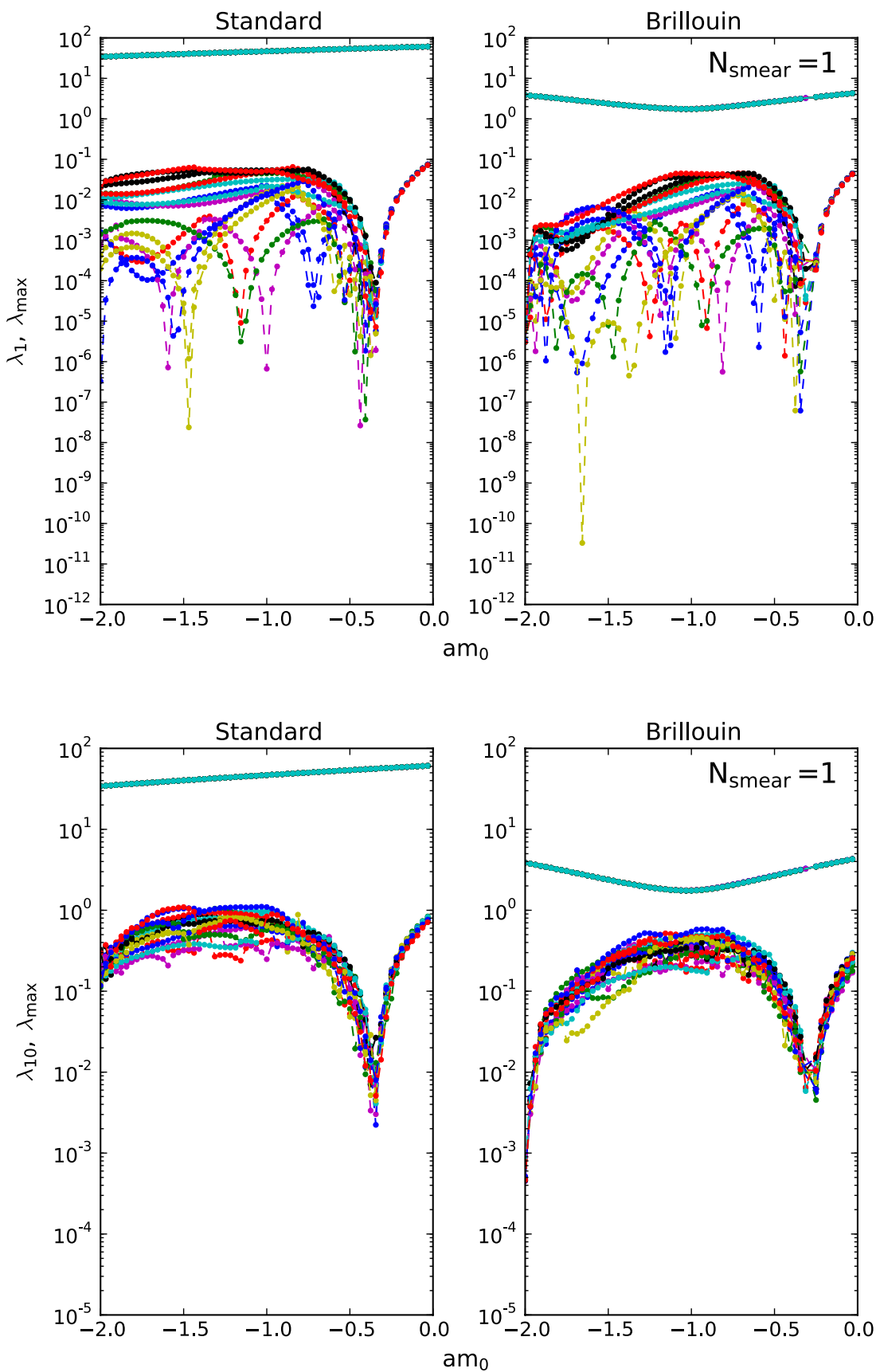

FIG. 19 (color online). The 1st (top), 10th (bottom), and largest Ritz eigenvalue of $D_{m}^{\dagger} D_{m}$ for the Wilson and the Brillouin operator, on 16 configurations at $\beta=6.20$, with $c_{\mathrm{SW}}=0$ and 1 step of APE smearing.

true that with the Brillouin operator the norm of the commutator is about an order of magnitude smaller than with the Wilson operator.

\section{Comparing the Ginsparg-Wilson violation of the kernels}

The Ginsparg-Wilson relation for a massless $D$ reads [10]

$$
\gamma_{5} D+D \gamma_{5}=\frac{a}{\rho} D \gamma_{5} D
$$

and we intend to plug in our operators with $\kappa$ set to $\kappa_{\text {crit }}$. Since the latter are known only for $c_{\mathrm{SW}}=1$ (cf. Table I) we do this with improvement. We measure $\|\left(D \gamma_{5}+\gamma_{5} D-\right.$ $\left.D \gamma_{5} D\right) \eta \|$ for a few dozen normalized Gaussian random vectors $\eta$ on 15 configurations of each ensemble. The result is shown in Fig. 18. A priori it is not clear whether it makes sense to plug a distinctly nonchiral operator into the Ginsparg-Wilson relation (27), but the result of our experiment seems to suggest that at least for the Brillouin operator it does. 

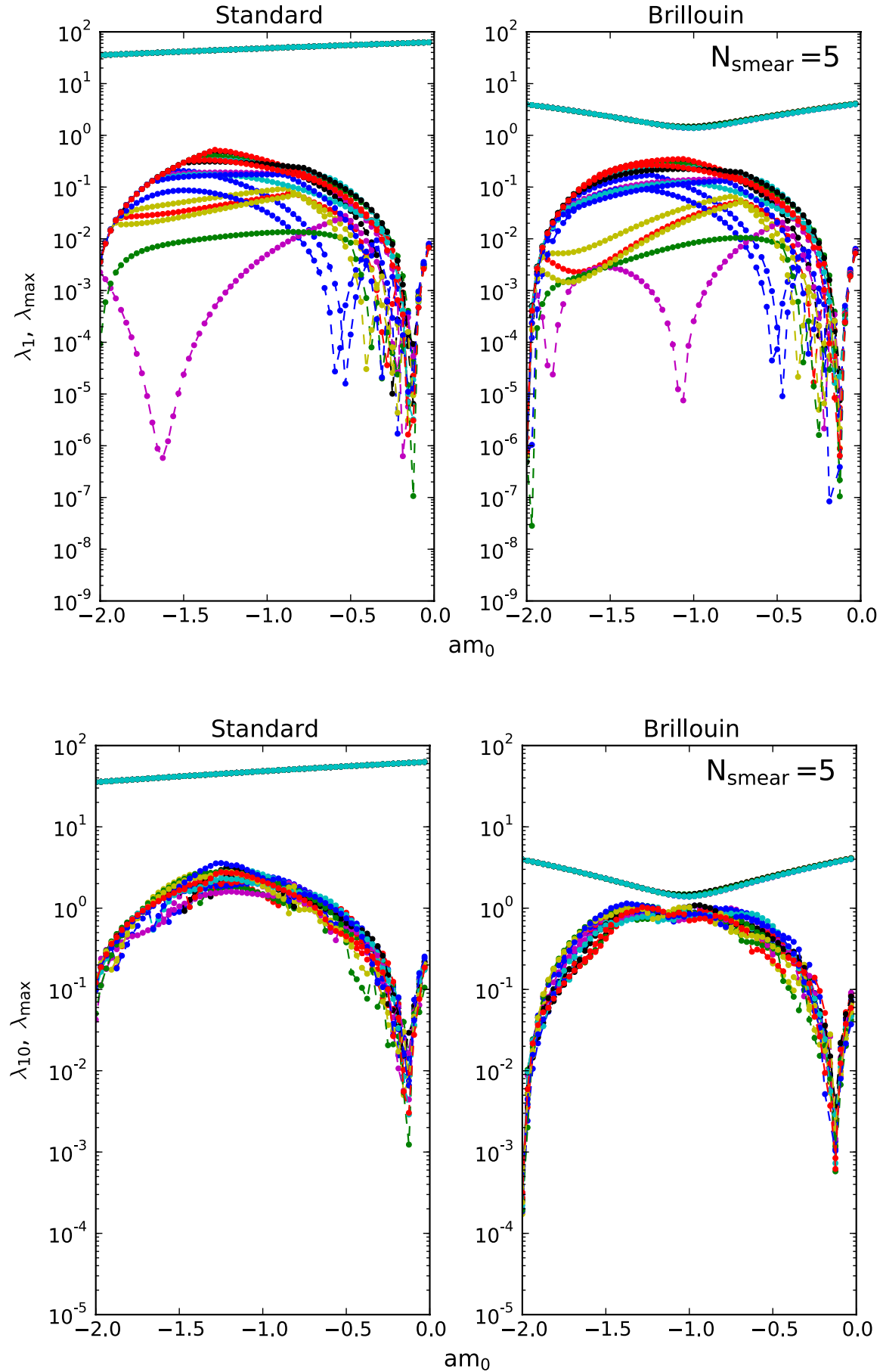

FIG. 20 (color online). The 1st (top), 10th (bottom), and largest Ritz eigenvalue of $D_{m}^{\dagger} D_{m}$ for the Wilson and the Brillouin operator, on 16 configurations at $\beta=6.20$, with $c_{\mathrm{SW}}=0$ and 5 steps of APE smearing.

\section{Comparing the condition number of the Hermitean kernels}

The cost of the overlap construction is determined by the smallest mode (in absolute magnitude) of the shifted Hermitean kernel $H_{\mathrm{kn},-\rho / a}=\gamma_{5} D_{\mathrm{kn},-\rho / a}=\gamma_{5}\left(D_{\mathrm{kn}}-\right.$ $\rho / a)$. Equivalently, one can look at the condition number of the squared operator $A=D_{\mathrm{kn},-\rho / a}^{\dagger} D_{\mathrm{kn},-\rho / a}$. In practice, one considers the so-called Ritz eigenvalues, i.e., the eigenvalues of the symmetric tridiagonal matrix that emerges from the Lanczos process on $A$. They approximate the extremal eigenvalues of $A$.

In the top panel of Fig. 19, we plot the smallest and the largest Ritz eigenvalue of $A$ made from the standard Wilson kernel (left) or the Brillouin kernel (right) as a function of the mass $a m_{0}=-\rho$. In the bottom panel, the 

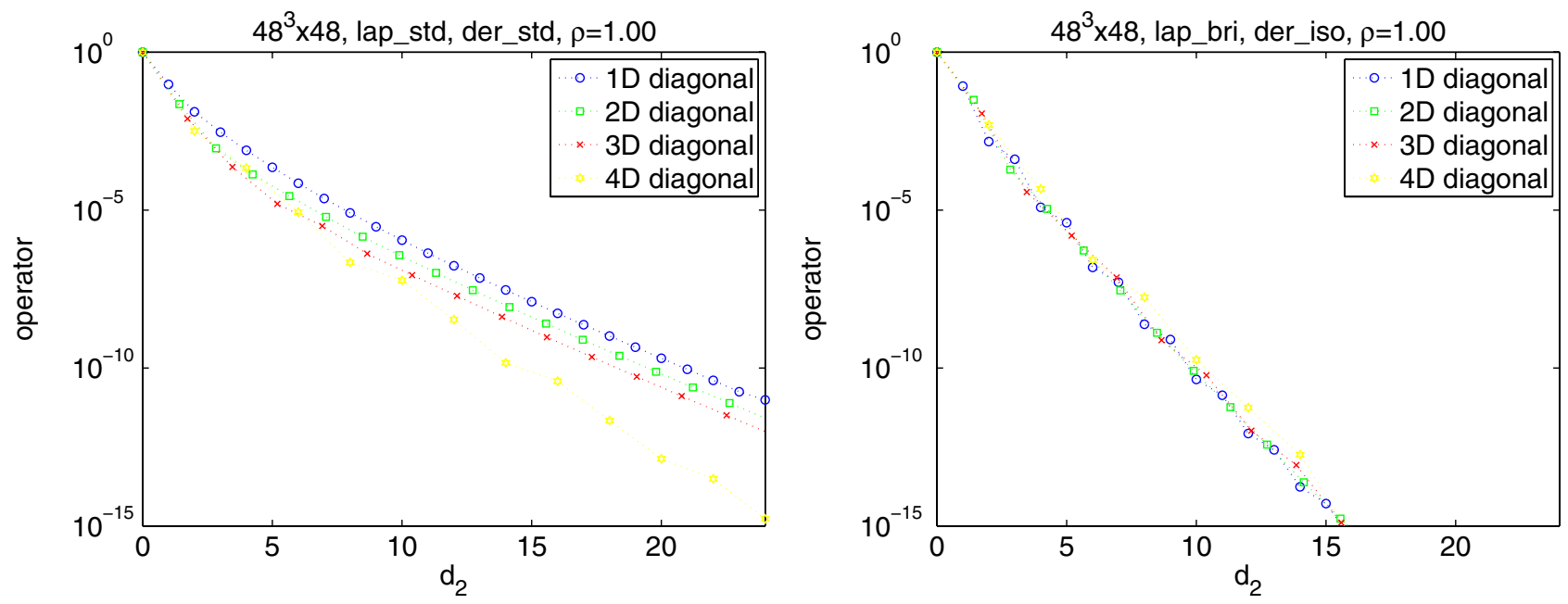

FIG. 21 (color online). Localization of the $\rho=1$ overlap operator with the standard Wilson kernel (left) or the new Brillouin kernel (right) on a free $48^{4}$ lattice, for four directions of the separation.

10th and (again) the largest eigenvalues are shown. In either panel, 16 configurations of our finest ensemble $(\beta=$ 6.20) are used, after 1 step of APE smearing is applied, and the clover coefficient is set to zero. Note that the gap between the largest and the smallest eigenvalue is just the condition number of $A$, and the gap between the largest and the 10th eigenvalue is the condition number of $A$ restricted to the subspace orthogonal to the lowest 9 eigenmodes. Hence, after $O(10)$ eigenmodes are projected, the Brillouin kernel allows for a reduced order of the polynomial or rational representation of the sign function, since its spectral range is 1-2 orders of magnitude smaller.

In Fig. 20 the same exercise is repeated with 5 steps of APE smearing. The overall picture is unchanged; again the resulting condition number of the Brillouin kernel is significantly smaller. We also find little impact of 19 (instead of 9) eigen modes projected, and whether the kernel is Symanzik improved or not. In short, the reduction of the condition number comes predominantly from the lowering of the largest eigenvalue (in line with what one would expect from the eigenvalue spectra shown in Fig. 8). By chance, one of the configurations used in Fig. 20 happens to be close (in configuration space) to a barrier between two topological sectors. With the Wilson kernel, the crossing occurs near $\rho=1.7$, with the Brillouin kernel close to $\rho=1.1$.

In summary, we find that the shifted Brillouin kernel has a significantly reduced condition number, in particular, with a bit of link smearing and after $O(10)$ eigen modes are projected. This allows for a lower degree polynomial or rational representation of the sign function.

\section{E. Comparing the locality of the resulting overlap actions}

The locality of the overlap action with standard Wilson kernel was first studied in [41]. In [46], it was shown that a nearly chiral (but still ultralocal) kernel can significantly improve the coordinate-space locality of the resulting overlap action. In $[42,43]$, it was shown that even a slight modification through some link smearing can lead to a considerable improvement. Therefore, one may hope that trading the Wilson kernel for the Brillouin kernel leads to a noticeable improvement of the locality of the overlap operator.

The localization of the overlap made from the Wilson or the Brillouin kernel is shown for a $48^{4}$ lattice in the freefield case in Fig. 21. The Frobenius norm of $D(x, y)$ as a function of the Euclidean distance $d_{2}=\|x-y\|_{2}$ is plotted. Evidently, the Brillouin kernel diminishes the anisotropy effects and makes the operator fall off at about twice the rate as before.

\section{SUMMARY}

We have introduced an ultralocal single-flavor lattice Dirac operator, based on the gauge covariant versions of $\nabla^{\text {iso }}$ and $\triangle^{\text {bri }}$ in (14). Relative to the Wilson operator, its eigenvalue spectrum is more Ginsparg-Wilson-like (cf. Fig. 22), and its dispersion relation is more continuum-like. ${ }^{8}$ As species doubling and global anomalies depend only on topological features of the dispersion relation $[48,49]$, from the conceptual viewpoint this is a Wilson-like fermion.

When combined with some link smearing and clover improvement, our action was found to show good scaling of decay constants even in the physical charm region, and we expect that the near agreement between perturbative and nonperturbative improvement coefficients found with the Wilson operator $[30,50,51]$ carries over to this action, too. It appears that lattice perturbation theory is conceptually not any more difficult than for standard Wilson

\footnotetext{
${ }^{8} \mathrm{~A}$ similar strategy has been adopted for staggered fermions in [47].
} 
$2 \mathrm{D}, \mathrm{U}(1): \mathrm{L}=24, \beta=3.3$

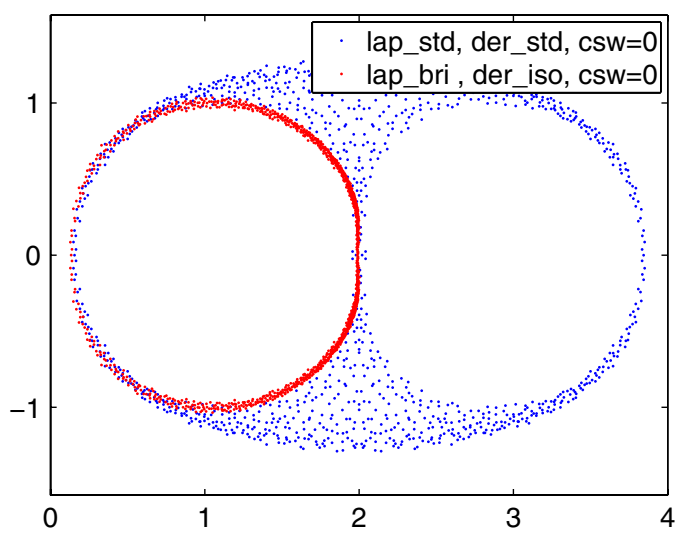

$4 \mathrm{D}, \mathrm{U}(1): \mathrm{L}=6, \beta=1.1$

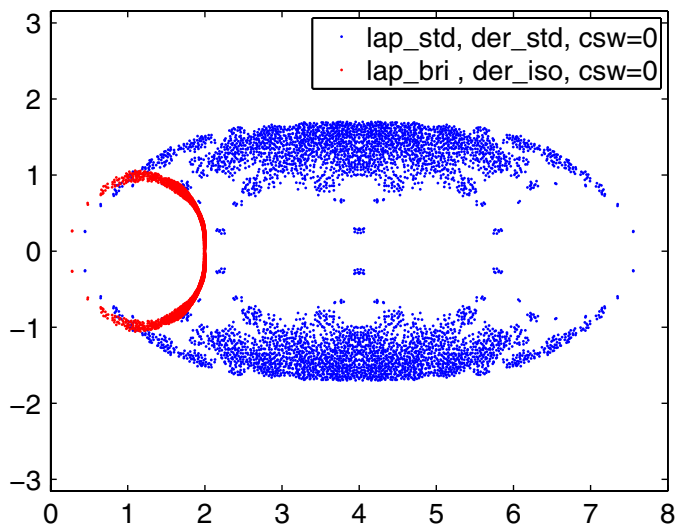

FIG. 22 (color online). Wilson (lap_std, der_std) and Brillouin (lap_bri, der_iso) eigenvalue spectra without link smearing and without clover improvement in 2D (left) and 4D (right).

fermions, but intermediate expressions may be longer, in particular, if several smearing steps are included and the backgrounds are made from improved glue [52].

Regarding the cost of a simulation with the Brillouin operator, it is hard to make generic statements. What can be compared is the number of forward applications needed (at a given value of $M_{\pi}$, cf. Fig. 15, where our Brillouin operator is seen to fare better). However, the cost of an individual forward application depends very much on the architecture used. One extreme case is a serial machine which is CPU limited; in this case 80 neighbor couplings instead of 8 make each application a factor $O(10)$ slower, whereupon the advantage is gone. On the other hand, highly threaded architectures such as graphics processing units (for an early application to lattice QCD see [53]) may be entirely bandwidth limited; in such a case, clever coding might keep the cost of a forward application essentially unchanged, relative to the Wilson operator. In our view, the upshot is that the usefulness of the Brillouin operator should be tested in phenomenological applications where all aspects of a formulation play a role, including the onset of the Symanzik scaling regime. In addition, there is a faint possibility that the Brillouin operator might be more susceptible to multigrid methods to solve for a given righthand vector.

Our Brillouin operator is a specific representative of the class of Dirac operators

$$
D(x, y)=\sum_{\mu} \gamma_{\mu} \rho_{\mu}(x-y)+\lambda(x-y),
$$

where the derivative and the Laplacian are expressed as

$$
\begin{gathered}
\rho_{\mu}(x-y)=\rho_{1}\left[\delta_{x+\hat{\mu}, y}-\delta_{x-\hat{\mu}, y}\right]+\rho_{2} \sum_{\nu}\left[\delta_{x+\hat{\mu}+\hat{\nu}, y}-\delta_{x-\hat{\mu}+\hat{\nu}, y}\right]+\rho_{3} \sum_{\nu, \rho}\left[\delta_{x+\hat{\mu}+\hat{\nu}+\hat{\rho}, y}-\delta_{x-\hat{\mu}+\hat{\nu}+\hat{\rho}, y}\right] \\
\quad+\rho_{4} \sum_{\nu, \rho, \sigma}\left[\delta_{x+\hat{\mu}+\hat{\nu}+\hat{\rho}+\hat{\sigma}, y}-\delta_{x-\hat{\mu}+\hat{\nu}+\hat{\rho}+\hat{\sigma}, y}\right] \\
\lambda(x-y)=\lambda_{0} \delta_{x, y}+\lambda_{1} \sum_{\mu}\left[\delta_{x+\hat{\mu}, y}-\delta_{x-\hat{\mu}, y}\right]+\lambda_{2} \sum_{\mu, \nu}\left[\delta_{x+\hat{\mu}+\hat{\nu}, y}-\delta_{x-\hat{\mu}+\hat{\nu}, y}\right]+\lambda_{3} \sum_{\mu, \nu, \rho}\left[\delta_{x+\hat{\mu}+\hat{\nu}+\hat{\rho}, y}-\delta_{x-\hat{\mu}+\hat{\nu}+\hat{\rho}, y]}\right. \\
+\lambda_{4} \sum_{\mu, \nu, \rho, \sigma}\left[\delta_{x+\hat{\mu}+\hat{\nu}+\hat{\rho}+\hat{\sigma}, y}-\delta_{x-\hat{\mu}+\hat{\nu}+\hat{\rho}+\hat{\sigma}, y}\right]
\end{gathered}
$$

with the understanding that the sums extend over positive and negative directions mutually orthogonal to each other (and in case of the derivative terms also to $\hat{\mu}$ ). As discussed in [54], in order to obtain the correct continuum dispersion relation one requires

$$
2 \rho_{1}+12 \rho_{2}+24 \rho_{3}+16 \rho_{4}=1, \quad \lambda_{0}+8 \lambda_{1}+24 \lambda_{2}+32 \lambda_{3}+16 \lambda_{4}=0
$$

which all operators in Table II obey. In addition, our Brillouin (der_iso, lap_bri) operator satisfies $\lambda_{0}+4 \lambda_{1}-16 \lambda_{3}-16 \lambda_{4}=2, \quad \lambda_{0}-8 \lambda_{2}+16 \lambda_{4}=2, \quad \lambda_{0}-4 \lambda_{1}+16 \lambda_{3}-16 \lambda_{4}=2, \quad \lambda_{0}-8 \lambda_{1}+24 \lambda_{2}-32 \lambda_{3}+16 \lambda_{4}=2$, 
TABLE II. The $\rho_{i}$ and $\lambda_{i}$ of the derivative and Laplacian parts of three Wilson-type fermions (Wilson's version, the hypercube fermion by Bietenholz et al. [14], and our Brillouin operator).

\begin{tabular}{lcccccc}
\hline \hline & Wilson & "Hypercube" & Der_iso & & Wilson & Hypercube \\
\hline$\ldots$ & $\ldots$ & $\ldots$ & $\ldots$ & $\lambda_{0}$ & 4 & 1.852720547 \\
$\rho_{1}$ & $1 / 2$ & 0.136846794 & $64 / 432$ & $\lambda_{1}$ & $-1 / 2$ & -0.060757866 \\
$\rho_{2}$ & 0 & 0.032077284 & $16 / 432$ & $\lambda_{2}$ & 0 & -0.030036032 \\
$\rho_{3}$ & 0 & 0.011058131 & $4 / 432$ & $\lambda_{3}$ & 0 & -0.015967620 \\
$\rho_{4}$ & 0 & 0.004748991 & $1 / 432$ & $\lambda_{4}$ & 0 & -0.008426812 \\
\hline \hline
\end{tabular}

which means that in the weak coupling limit all doublers are lifted by an equal amount, and

$$
12 \rho_{2}+48 \rho_{3}+48 \rho_{4}-1=0,
$$

which ensures that the physical branch of the free-field dispersion relation $E / p$ has no $O\left(a^{2}\right)$ contribution [54] and that the leading cutoff effects in the deviation of the pressure from the Stefan-Boltzmann limit are $\propto 1 / N_{t}^{4}$ [54]. In other words, our Brillouin operator is expected to define a discretized version of QCD with decent bulk thermodynamic properties.

\section{ACKNOWLEDGMENTS}

We thank Zoltan Fodor for useful correspondence. Computations were performed on the high-performance cluster JUROPA at JSC, with resources allocated through a NIC grant. Both authors were supported by the SFB TR-55.

\section{APPENDIX A: DISCRETE LAPLACIANS AND DERIVATIVES IN 2D}

We give the following four Laplace stencils in 2D along with their momentum-space representation:

(i) standard Laplacian in 2D:

$$
\left[\begin{array}{ccc}
0 & 1 & 0 \\
1 & -4 & 1 \\
0 & 1 & 0
\end{array}\right] / 1
$$

$\hat{\triangle}=2 \cos \left(k_{1}\right)+2 \cos \left(k_{2}\right)-4$ [to be read as $\left.a^{2} \hat{\triangle}=2 \cos \left(a p_{1}\right)+2 \cos \left(a p_{2}\right)-4\right]$

(ii) tilted Laplacian in $2 \mathrm{D}$ :

$$
\left[\begin{array}{ccc}
1 & 0 & 1 \\
0 & -4 & 0 \\
1 & 0 & 1
\end{array}\right] / 2
$$

$\hat{\triangle}=2 \cos \left(k_{1}\right) \cos \left(k_{2}\right)-2$ [mind the second zero at $\left.k_{1}=k_{2}=\pi\right]$

(iii) Brillouin Laplacian in 2D:

$$
\left[\begin{array}{ccc}
1 & 2 & 1 \\
2 & -12 & 2 \\
1 & 2 & 1
\end{array}\right] / 4
$$

$\hat{\triangle}=4 \cos ^{2}\left(k_{1} / 2\right) \cos ^{2}\left(k_{2} / 2\right)-4$ [takes constant value -4 at boundary of Brillouin zone (BZ)]

(iv) isotropic Laplacian in 2D:

$$
\left[\begin{array}{ccc}
1 & 4 & 1 \\
4 & -20 & 4 \\
1 & 4 & 1
\end{array}\right] / 6
$$

$\hat{\triangle}=\left[2 \cos \left(k_{1}\right) \cos \left(k_{2}\right)+4 \cos \left(k_{1}\right)+4 \cos \left(k_{2}\right)-\right.$

$10] / 3$ [also know as the "Mehrstellen" Laplacian].

We give the following three $x$-derivative stencils in 2D along with their momentum-space representation:

(v) standard $x$-derivative in 2D:

$$
\left[\begin{array}{ccc}
0 & 0 & 0 \\
-1 & 0 & 1 \\
0 & 0 & 0
\end{array}\right] / 2
$$

$\hat{\partial}_{x}=\mathrm{i} \sin \left(k_{1}\right)$ [to be read as $a \hat{\partial}_{x}=\mathrm{i} \sin \left(a p_{1}\right)$ ]

(vi) Brillouin $x$-derivative in $2 \mathrm{D}$ :

$$
\left[\begin{array}{lll}
-1 & 0 & 1 \\
-2 & 0 & 2 \\
-1 & 0 & 1
\end{array}\right] / 8
$$

$\hat{\partial}_{x}=\mathrm{i} \sin \left(k_{1}\right)\left[\cos \left(k_{2}\right)+1\right] / 2$

(vii) isotropic $x$-derivative in 2D:

$$
\left[\begin{array}{lll}
-1 & 0 & 1 \\
-4 & 0 & 4 \\
-1 & 0 & 1
\end{array}\right] / 12
$$

$\hat{\partial}_{x}=\mathrm{i} \sin \left(k_{1}\right)\left[\cos \left(k_{2}\right)+2\right] / 3$.

\section{APPENDIX B: DISCRETE LAPLACIANS AND DERIVATIVES IN 3D}

We give the following four Laplace stencils in 3D along with their momentum-space representation:

(i) standard Laplacian in 3D:

$$
\begin{gathered}
{\left[\begin{array}{lll}
0 & 0 & 0 \\
0 & 1 & 0 \\
0 & 0 & 0
\end{array}\right]\left[\begin{array}{ccc}
0 & 1 & 0 \\
1 & -6 & 1 \\
0 & 1 & 0
\end{array}\right]\left[\begin{array}{lll}
0 & 0 & 0 \\
0 & 1 & 0 \\
0 & 0 & 0
\end{array}\right] / 1} \\
\widehat{\triangle}=2 \cos \left(k_{1}\right)+2 \cos \left(k_{2}\right)+2 \cos \left(k_{3}\right)-6
\end{gathered}
$$


(ii) tilted Laplacian in 3D:

$$
\left[\begin{array}{lll}
1 & 0 & 1 \\
0 & 0 & 0 \\
1 & 0 & 1
\end{array}\right]\left[\begin{array}{ccc}
0 & 0 & 0 \\
0 & -8 & 0 \\
0 & 0 & 0
\end{array}\right]\left[\begin{array}{lll}
1 & 0 & 1 \\
0 & 0 & 0 \\
1 & 0 & 1
\end{array}\right] / 4
$$

$\hat{\triangle}=2 \cos \left(k_{1}\right) \cos \left(k_{2}\right) \cos \left(k_{3}\right)-2$ [mind the three additional zeros]

(iii) Brillouin Laplacian in 3D:

$$
\left[\begin{array}{lll}
1 & 2 & 1 \\
2 & 4 & 2 \\
1 & 2 & 1
\end{array}\right]\left[\begin{array}{ccc}
2 & 4 & 2 \\
4 & -56 & 4 \\
2 & 4 & 2
\end{array}\right]\left[\begin{array}{lll}
1 & 2 & 1 \\
2 & 4 & 2 \\
1 & 2 & 1
\end{array}\right] / 16
$$

$\hat{\triangle}=4 \cos ^{2}\left(k_{1} / 2\right) \cos ^{2}\left(k_{2} / 2\right) \cos ^{2}\left(k_{3} / 2\right)-4$ [takes constant value at boundary of BZ]

(iv) isotropic Laplacian in 3D:

$$
\begin{aligned}
& {\left[\begin{array}{ccc}
1 & 6 & 1 \\
6 & 20 & 6 \\
1 & 6 & 1
\end{array}\right]\left[\begin{array}{ccc}
6 & 20 & 6 \\
20 & -200 & 20 \\
6 & 20 & 6
\end{array}\right]\left[\begin{array}{ccc}
1 & 6 & 1 \\
6 & 20 & 6 \\
1 & 6 & 1
\end{array}\right] / 48} \\
& \hat{\triangle}=\left[\cos \left(k_{1}\right) \cos \left(k_{2}\right) \cos \left(k_{3}\right)+3 \cos \left(k_{1}\right) \cos \left(k_{2}\right)+\ldots\right. \\
& \left.+5 \cos \left(k_{1}\right)+\ldots-25\right] / 6 .
\end{aligned}
$$

We give the following three $x$-derivative stencils in 3D along with their momentum-space representation:

(v) standard $x$-derivative in 3D:

$$
\left[\begin{array}{lll}
0 & 0 & 0 \\
0 & 0 & 0 \\
0 & 0 & 0
\end{array}\right]\left[\begin{array}{ccc}
0 & 0 & 0 \\
-1 & 0 & 1 \\
0 & 0 & 0
\end{array}\right]\left[\begin{array}{lll}
0 & 0 & 0 \\
0 & 0 & 0 \\
0 & 0 & 0
\end{array}\right] / 2
$$

$\hat{\partial}_{x}=\mathrm{i} \sin \left(k_{1}\right)$

(vi) Brillouin $x$-derivative in 3D:

$$
\left[\begin{array}{lll}
-1 & 0 & 1 \\
-2 & 0 & 2 \\
-1 & 0 & 1
\end{array}\right]\left[\begin{array}{lll}
-2 & 0 & 2 \\
-4 & 0 & 4 \\
-2 & 0 & 2
\end{array}\right]\left[\begin{array}{lll}
-1 & 0 & 1 \\
-2 & 0 & 2 \\
-1 & 0 & 1
\end{array}\right] / 32
$$

$\hat{\partial}_{x}=\mathrm{i} \sin \left(k_{1}\right)\left[\cos \left(k_{2}\right)+1\right]\left[\cos \left(k_{3}\right)+1\right] / 4$

(vii) isotropic $x$-derivative in $3 \mathrm{D}$ :

$$
\left[\begin{array}{ccc}
-1 & 0 & 1 \\
-4 & 0 & 4 \\
-1 & 0 & 1
\end{array}\right]\left[\begin{array}{ccc}
-4 & 0 & 4 \\
-16 & 0 & 16 \\
-4 & 0 & 4
\end{array}\right]\left[\begin{array}{ccc}
-1 & 0 & 1 \\
-4 & 0 & 4 \\
-1 & 0 & 1
\end{array}\right] / 72
$$

$\hat{\partial}_{x}=\mathrm{i} \sin \left(k_{1}\right)\left[\cos \left(k_{2}\right)+2\right]\left[\cos \left(k_{3}\right)+2\right] / 9$.

\section{APPENDIX C: DISCRETE LAPLACIANS AND DERIVATIVES IN 4D}

We give the following four Laplace stencils in 4D along with their momentum-space representation: (i) standard Laplacian in 4D:

$$
\begin{aligned}
& {\left[\begin{array}{lll}
0 & 0 & 0 \\
0 & 0 & 0 \\
0 & 0 & 0
\end{array}\right]\left[\begin{array}{lll}
0 & 0 & 0 \\
0 & 1 & 0 \\
0 & 0 & 0
\end{array}\right]\left[\begin{array}{lll}
0 & 0 & 0 \\
0 & 0 & 0 \\
0 & 0 & 0
\end{array}\right]} \\
& {\left[\begin{array}{lll}
0 & 0 & 0 \\
0 & 1 & 0 \\
0 & 0 & 0
\end{array}\right]\left[\begin{array}{ccc}
0 & 1 & 0 \\
1 & -8 & 1 \\
0 & 1 & 0
\end{array}\right]\left[\begin{array}{lll}
0 & 0 & 0 \\
0 & 1 & 0 \\
0 & 0 & 0
\end{array}\right] / 1} \\
& {\left[\begin{array}{lll}
0 & 0 & 0 \\
0 & 0 & 0 \\
0 & 0 & 0
\end{array}\right]\left[\begin{array}{lll}
0 & 0 & 0 \\
0 & 1 & 0 \\
0 & 0 & 0
\end{array}\right]\left[\begin{array}{lll}
0 & 0 & 0 \\
0 & 0 & 0 \\
0 & 0 & 0
\end{array}\right]}
\end{aligned}
$$

$$
\hat{\triangle}=2 \cos \left(k_{1}\right)+2 \cos \left(k_{2}\right)+2 \cos \left(k_{3}\right)+2 \cos \left(k_{4}\right)-8
$$

(ii) tilted Laplacian in $4 \mathrm{D}$ :

$$
\begin{aligned}
& {\left[\begin{array}{lll}
1 & 0 & 1 \\
0 & 0 & 0 \\
1 & 0 & 1
\end{array}\right]\left[\begin{array}{lll}
0 & 0 & 0 \\
0 & 0 & 0 \\
0 & 0 & 0
\end{array}\right]\left[\begin{array}{lll}
1 & 0 & 1 \\
0 & 0 & 0 \\
1 & 0 & 1
\end{array}\right]} \\
& {\left[\begin{array}{lll}
0 & 0 & 0 \\
0 & 0 & 0 \\
0 & 0 & 0
\end{array}\right]\left[\begin{array}{ccc}
0 & 0 & 0 \\
0 & -16 & 0 \\
0 & 0 & 0
\end{array}\right]\left[\begin{array}{lll}
0 & 0 & 0 \\
0 & 0 & 0 \\
0 & 0 & 0
\end{array}\right] / 8} \\
& {\left[\begin{array}{lll}
1 & 0 & 1 \\
0 & 0 & 0 \\
1 & 0 & 1
\end{array}\right]\left[\begin{array}{lll}
0 & 0 & 0 \\
0 & 0 & 0 \\
0 & 0 & 0
\end{array}\right]\left[\begin{array}{lll}
1 & 0 & 1 \\
0 & 0 & 0 \\
1 & 0 & 1
\end{array}\right]}
\end{aligned}
$$

$\hat{\triangle}=2 \cos \left(k_{1}\right) \cos \left(k_{2}\right) \cos \left(k_{3}\right) \cos \left(k_{4}\right)-2$ [mind the seven additional zeros]

(iii) Brillouin Laplacian in 4D:

$$
\begin{aligned}
& {\left[\begin{array}{lll}
1 & 2 & 1 \\
2 & 4 & 2 \\
1 & 2 & 1
\end{array}\right]\left[\begin{array}{lll}
2 & 4 & 2 \\
4 & 8 & 4 \\
2 & 4 & 2
\end{array}\right]\left[\begin{array}{lll}
1 & 2 & 1 \\
2 & 4 & 2 \\
1 & 2 & 1
\end{array}\right]} \\
& {\left[\begin{array}{lll}
2 & 4 & 2 \\
4 & 8 & 4 \\
2 & 4 & 2
\end{array}\right]\left[\begin{array}{ccc}
4 & 8 & 4 \\
8 & -240 & 8 \\
4 & 8 & 4
\end{array}\right]\left[\begin{array}{lll}
2 & 4 & 2 \\
4 & 8 & 4 \\
2 & 4 & 2
\end{array}\right] / 64} \\
& {\left[\begin{array}{lll}
1 & 2 & 1 \\
2 & 4 & 2 \\
1 & 2 & 1
\end{array}\right]\left[\begin{array}{lll}
2 & 4 & 2 \\
4 & 8 & 4 \\
2 & 4 & 2
\end{array}\right]\left[\begin{array}{lll}
1 & 2 & 1 \\
2 & 4 & 2 \\
1 & 2 & 1
\end{array}\right]}
\end{aligned}
$$

$\hat{\triangle}=4 \cos ^{2}\left(k_{1} / 2\right) \cos ^{2}\left(k_{2} / 2\right) \cos ^{2}\left(k_{3} / 2\right) \cos ^{2}\left(k_{4} / 2\right)-$ 4 [constant at boundary of BZ] 
(iv) isotropic Laplacian in 4D:

$\left[\begin{array}{lcc}1 & 7 & 1 \\ 7 & 40 & 7 \\ 1 & 7 & 1\end{array}\right]\left[\begin{array}{ccc}7 & 40 & 7 \\ 40 & 100 & 40 \\ 7 & 40 & 7\end{array}\right]\left[\begin{array}{ccc}1 & 7 & 1 \\ 7 & 40 & 7 \\ 1 & 7 & 1\end{array}\right]$

$\left[\begin{array}{ccc}7 & 40 & 7 \\ 40 & 100 & 40 \\ 7 & 40 & 7\end{array}\right]\left[\begin{array}{ccc}40 & 100 & 40 \\ 100 & -2000 & 100 \\ 40 & 100 & 40\end{array}\right]\left[\begin{array}{ccc}7 & 40 & 7 \\ 40 & 100 & 40 \\ 7 & 40 & 7\end{array}\right] / 432$

$\left[\begin{array}{lcc}1 & 7 & 1 \\ 7 & 40 & 7 \\ 1 & 7 & 1\end{array}\right]\left[\begin{array}{ccc}7 & 40 & 7 \\ 40 & 100 & 40 \\ 7 & 40 & 7\end{array}\right]\left[\begin{array}{ccc}1 & 7 & 1 \\ 7 & 40 & 7 \\ 1 & 7 & 1\end{array}\right]$

$\hat{\triangle}=\left[2 c_{1} c_{2} c_{3} c_{4}+7 c_{1} c_{2} c_{3}+\ldots+20 c_{1} c_{2}+\ldots+\right.$

$\left.25 c_{1}+\ldots-250\right] / 54$ [with $c_{1}=\cos \left(k_{1}\right)$, etc.].

We give the following three $x$-derivative stencils in $4 \mathrm{D}$ along with their momentum-space representation:

(v) standard $x$-derivative in $4 \mathrm{D}$ :

$$
\begin{aligned}
& {\left[\begin{array}{lll}
0 & 0 & 0 \\
0 & 0 & 0 \\
0 & 0 & 0
\end{array}\right]\left[\begin{array}{lll}
0 & 0 & 0 \\
0 & 0 & 0 \\
0 & 0 & 0
\end{array}\right]\left[\begin{array}{lll}
0 & 0 & 0 \\
0 & 0 & 0 \\
0 & 0 & 0
\end{array}\right]} \\
& {\left[\begin{array}{lll}
0 & 0 & 0 \\
0 & 0 & 0 \\
0 & 0 & 0
\end{array}\right]\left[\begin{array}{ccc}
0 & 0 & 0 \\
-1 & 0 & 1 \\
0 & 0 & 0
\end{array}\right]\left[\begin{array}{lll}
0 & 0 & 0 \\
0 & 0 & 0 \\
0 & 0 & 0
\end{array}\right] / 2} \\
& {\left[\begin{array}{lll}
0 & 0 & 0 \\
0 & 0 & 0 \\
0 & 0 & 0
\end{array}\right]\left[\begin{array}{lll}
0 & 0 & 0 \\
0 & 0 & 0 \\
0 & 0 & 0
\end{array}\right]\left[\begin{array}{lll}
0 & 0 & 0 \\
0 & 0 & 0 \\
0 & 0 & 0
\end{array}\right]}
\end{aligned}
$$

$\hat{\partial}_{x}=\mathrm{i} \sin \left(k_{1}\right)$

(vi) Brillouin $x$-derivative in 4D:

$$
\begin{aligned}
& {\left[\begin{array}{lll}
-1 & 0 & 1 \\
-2 & 0 & 2 \\
-1 & 0 & 1
\end{array}\right]\left[\begin{array}{lll}
-2 & 0 & 2 \\
-4 & 0 & 4 \\
-2 & 0 & 2
\end{array}\right]\left[\begin{array}{lll}
-1 & 0 & 1 \\
-2 & 0 & 2 \\
-1 & 0 & 1
\end{array}\right]} \\
& {\left[\begin{array}{lll}
-2 & 0 & 2 \\
-4 & 0 & 4 \\
-2 & 0 & 2
\end{array}\right]\left[\begin{array}{lll}
-4 & 0 & 4 \\
-8 & 0 & 8 \\
-4 & 0 & 4
\end{array}\right]\left[\begin{array}{lll}
-2 & 0 & 2 \\
-4 & 0 & 4 \\
-2 & 0 & 2
\end{array}\right] / 128} \\
& {\left[\begin{array}{lll}
-1 & 0 & 1 \\
-2 & 0 & 2 \\
-1 & 0 & 1
\end{array}\right]\left[\begin{array}{lll}
-2 & 0 & 2 \\
-4 & 0 & 4 \\
-2 & 0 & 2
\end{array}\right]\left[\begin{array}{lll}
-1 & 0 & 1 \\
-2 & 0 & 2 \\
-1 & 0 & 1
\end{array}\right]}
\end{aligned}
$$$$
\hat{\partial}_{x}=\mathrm{i} \sin \left(k_{1}\right)\left[\cos \left(k_{2}\right)+1\right]\left[\cos \left(k_{3}\right)+1\right]\left[\cos \left(k_{4}\right)+1\right] / 8
$$

(vii) isotropic $x$-derivative in 4D:

$$
\begin{aligned}
& {\left[\begin{array}{lll}
-1 & 0 & 1 \\
-4 & 0 & 4 \\
-1 & 0 & 1
\end{array}\right]\left[\begin{array}{ccc}
-4 & 0 & 4 \\
-16 & 0 & 16 \\
-4 & 0 & 4
\end{array}\right]\left[\begin{array}{llc}
-1 & 0 & 1 \\
-4 & 0 & 4 \\
-1 & 0 & 1
\end{array}\right] } \\
& {\left[\begin{array}{ccc}
-4 & 0 & 4 \\
-16 & 0 & 16 \\
-4 & 0 & 4
\end{array}\right]\left[\begin{array}{ccc}
-16 & 0 & 16 \\
-64 & 0 & 64 \\
-16 & 0 & 16
\end{array}\right]\left[\begin{array}{ccc}
-4 & 0 & 4 \\
-16 & 0 & 16 \\
-4 & 0 & 4
\end{array}\right] / 432 } \\
& {\left[\begin{array}{lll}
-1 & 0 & 1 \\
-4 & 0 & 4 \\
-1 & 0 & 1
\end{array}\right]\left[\begin{array}{ccc}
-4 & 0 & 4 \\
-16 & 0 & 16 \\
-4 & 0 & 4
\end{array}\right]\left[\begin{array}{ccc}
-1 & 0 & 1 \\
-4 & 0 & 4 \\
-1 & 0 & 1
\end{array}\right] } \\
\hat{\partial}_{x}= & \mathrm{i} \sin \left(k_{1}\right)\left[\cos \left(k_{2}\right)+2\right]\left[\cos \left(k_{3}\right)+2\right]\left[\cos \left(k_{4}\right)+2\right] / 27 .
\end{aligned}
$$

\section{APPENDIX D: ISOTROPIC STENCILS VIA KUMAR'S TRICK}

A somewhat systematic overview that includes isotropic Laplacians in 2D and 3D is presented in [21]. In this Appendix, we review a particularly practical approach for deriving an isotropic stencil in any dimension due to Kumar [20].

The standard discretization of the first derivative operator in $2 \mathrm{D}$ is

$$
\left(\psi_{x}\right)_{i, j}^{\mathrm{std}}=\frac{1}{2 a}\left(\psi_{i+1, j}-\psi_{i-1, j}\right) .
$$

From a Taylor expansion [of i $\sin \left(a k_{1}\right)=\mathrm{i} a k_{1}\left(1-a^{2} k_{1}^{2} /\right.$ $\left.6+O\left(a^{4}\right)\right)$ in momentum space or directly in position space] one finds that the standard discrete derivative deviates from the continuum derivative through $O\left(a^{2}\right)$-suppressed terms. This may be summarized in the form

$$
\left(\psi_{x}\right)_{i, j}^{\mathrm{std}}=\left(1+\frac{a^{2}}{6} \partial_{x x}\right)\left(\psi_{x}\right)_{i, j},
$$

where both derivatives on the right-hand side (rhs) refer to the continuum. A possible strategy to improve rotational symmetry is thus to define the discretized first derivative such that the deviation from the continuum behavior i $a k_{1}$ is the same in either direction that is through

$$
\left(\psi_{x}\right)_{i, j}^{\text {iso }}=\left(1+\frac{a^{2}}{6} \triangle\right)\left(\psi_{x}\right)_{i, j},
$$

where again the operators on the rhs refer to the continuum. The idea by Kumar [20] is to factor the bracket and to define the discretized isotropic first derivative through

$$
\begin{aligned}
\left(\psi_{x}\right)_{i, j}^{\text {iso }} & =\left(1+\frac{a^{2}}{6} \partial_{y y}\right)\left(1+\frac{a^{2}}{6} \partial_{x x}\right)\left(\psi_{x}\right)_{i, j} \\
& =\left(1+\frac{a^{2}}{6} \partial_{y y}\right)\left(\psi_{x}\right)_{i, j}^{\mathrm{std}},
\end{aligned}
$$


where (D2) has been used in the second step. Moreover, we may replace the second derivative in the $y$ direction by its simplest discrete version (i.e., the $1 /-2 / 1$ stencil operator), since the difference is another $a^{4}$ term [of which we did not keep track in (D2)-(D4) anyway]. This gives

$$
\begin{aligned}
\left(\psi_{x}\right)_{i, j}^{\text {iso }}= & {\left[\begin{array}{ccc}
0 & 0 & 0 \\
-1 / 2 & 0 & -1 / 2 \\
0 & 0 & 0
\end{array}\right]+\frac{1}{6}\left[\begin{array}{ccc}
-1 / 2 & 0 & 1 / 2 \\
1 & 0 & -1 \\
-1 / 2 & 0 & 1 / 2
\end{array}\right] } \\
= & {\left[\begin{array}{ccc}
-1 & 0 & 1 \\
-4 & 0 & 4 \\
-1 & 0 & 1
\end{array}\right] / 12 } \\
= & {\left[\psi_{i+1, j+1}+4 \psi_{i+1, j}+\psi_{i+1, j-1}-\psi_{i-1, j+1}\right.} \\
& \left.-4 \psi_{i-1, j}-\psi_{i-1, j-1}\right] / 12,
\end{aligned}
$$

where we have used the stencil notation. Compared to the standard discrete derivative, there is a spreading in the transverse direction with a factor $\frac{1}{6} / \frac{4}{6} / \frac{1}{6}$, respectively. It is easy to generalize this procedure to higher dimensions, and the pertinent isotropic first derivative operators have been given in Appendices A, B, and C for 2D, 3D, 4D, respectively.

The standard discretization of the second derivative operator in $2 \mathrm{D}$ is

$$
\left(\psi_{x x}\right)_{i, j}^{\mathrm{std}}=\frac{1}{a^{2}}\left(\psi_{i+1, j}-2 \psi_{i, j}+\psi_{i-1, j}\right)
$$

and from a Taylor expansion one finds that this is equivalent to

$$
\left(\psi_{x x}\right)_{i, j}^{\mathrm{std}}=\left(1+\frac{a^{2}}{12} \partial_{x x}\right)\left(\psi_{x x}\right)_{i, j},
$$

where both derivatives on the rhs refer to the continuum. The isotropic second derivative operator follows by deliberately introducing the same discretization error in the $y$-direction

$$
\left(\psi_{x x}\right)_{i, j}^{\text {iso }}=\left(1+\frac{a^{2}}{12} \triangle\right)\left(\psi_{x x}\right)_{i, j}
$$

and Kumar's trick [20] of factorizing (to the order we are interested in) the continuum expression

$$
\begin{aligned}
\left(\psi_{x x}\right)_{i, j}^{\text {iso }}= & \left(1+\frac{a^{2}}{12} \partial_{y y}\right)\left(1+\frac{a^{2}}{12} \partial_{x x}\right)\left(\psi_{x x}\right)_{i, j} \\
= & \left(1+\frac{a^{2}}{12} \partial_{y y}\right)\left(\psi_{x x}\right)_{i, j}^{\text {std }} \\
= & {\left[\psi_{i+1, j+1}+10 \psi_{i+1, j}+\psi_{i+1, j-1}\right.} \\
& -2 \psi_{i, j+1}-20 \psi_{i, j}-2 \psi_{i, j-1}+\psi_{i-1, j+1} \\
& \left.+10 \psi_{i-1, j}+\psi_{i-1, j-1}\right] / 12
\end{aligned}
$$

yields (the simplest) isotropic second derivative operator. Compared to the standard discrete second derivative in 2D, there is a spreading in the transverse direction by a factor of $\frac{1}{12} / \frac{10}{12} / \frac{1}{12}$. It is easy to generalize this procedure to higher dimensions, and we shall just give the following results:

(i) isotropic second $x$-derivative in $2 \mathrm{D}$ :

$$
\left[\begin{array}{ccc}
1 & -2 & 1 \\
10 & -20 & 10 \\
1 & -2 & 1
\end{array}\right] / 12
$$

$$
\hat{\partial}_{x}^{2}=\left[\cos \left(k_{1}\right)-1\right]\left[\cos \left(k_{2}\right)+5\right] / 3
$$

(ii) isotropic second $x$-derivative in $3 \mathrm{D}$ :

$$
\begin{aligned}
& {\left[\begin{array}{ccc}
1 & -2 & 1 \\
10 & -20 & 10 \\
1 & -2 & 1
\end{array}\right]\left[\begin{array}{ccc}
10 & -20 & 10 \\
100 & -200 & 100 \\
10 & -20 & 10
\end{array}\right] } \\
& {\left[\begin{array}{ccc}
1 & -2 & 1 \\
10 & -20 & 10 \\
1 & -2 & 1
\end{array}\right] / 144 } \\
\hat{\partial}_{x}^{2}= & {\left[\cos \left(k_{1}\right)-1\right]\left[\cos \left(k_{2}\right)+5\right]\left[\cos \left(k_{3}\right)+5\right] / 18 }
\end{aligned}
$$

(iii) isotropic second $x$-derivative in 4D:

$$
\begin{aligned}
& {\left[\begin{array}{ccc}
1 & -2 & 1 \\
10 & -20 & 10 \\
1 & -2 & 1
\end{array}\right]\left[\begin{array}{ccc}
10 & -20 & 10 \\
100 & -200 & 100 \\
10 & -20 & 10
\end{array}\right]\left[\begin{array}{ccc}
1 & -2 & 1 \\
10 & -20 & 10 \\
1 & -2 & 1
\end{array}\right] } \\
& {\left[\begin{array}{ccc}
10 & -20 & 10 \\
100 & -200 & 100 \\
10 & -20 & 10
\end{array}\right]\left[\begin{array}{ccc}
100 & -200 & 100 \\
1000 & -2000 & 1000 \\
100 & -200 & 100
\end{array}\right]\left[\begin{array}{ccc}
10 & -20 & 10 \\
100 & -200 & 100 \\
10 & -20 & 10
\end{array}\right] / 1728 } \\
& {\left[\begin{array}{ccc}
1 & -2 & 1 \\
10 & -20 & 10 \\
1 & -2 & 1
\end{array}\right]\left[\begin{array}{ccc}
10 & -20 & 10 \\
100 & -200 & 100 \\
10 & -20 & 10
\end{array}\right]\left[\begin{array}{ccc}
1 & -2 & 1 \\
10 & -20 & 10 \\
1 & -2 & 1
\end{array}\right] } \\
& \hat{\partial}_{x}^{2}=\left[\cos \left(k_{1}\right)-1\right]\left[\cos \left(k_{2}\right)+5\right]\left[\cos \left(k_{3}\right)+5\right]\left[\cos \left(k_{4}\right)+5\right] / 108 .
\end{aligned}
$$


Upon adding the isotropic second derivative in the $y$ (and possibly $z, t$ ) direction, one gets the isotropic Laplacian stencil in $2 \mathrm{D}, 3 \mathrm{D}, 4 \mathrm{D}$, as given in previous Appendices. We emphasize that the isotropic Laplacian establishes better rotational symmetry near the center of the Brillouin zone. What proves most useful in many applications (including our goal of designing more continuum-like lattice Dirac operators), however, is isotropy at the boundary of the Brillouin zone, and this is achieved through the Brillouin Laplacian, as given in Appendices A, B, and C.
[1] K. G. Wilson, Phys. Rev. D 10, 2445 (1974).

[2] K. G. Wilson, New Phenomena in Subnuclear Physics, Part A. Proceedings of the First Half of the 1975 International School of Subnuclear Physics, Erice, Sicily, 1975, edited by A. Zichichi (Plenum Press, New York, 1977), p. 69.

[3] H. Neuberger, Phys. Lett. B 417, 141 (1998).

[4] H. Neuberger, Phys. Lett. B 427, 353 (1998).

[5] B. Sheikholeslami and R. Wohlert, Nucl. Phys. B259, 572 (1985).

[6] G. Heatlie, G. Martinelli, C. Pittori, G. C. Rossi, and C. T. Sachrajda, Nucl. Phys. B352, 266 (1991).

[7] G. Martinelli, C. T. Sachrajda, and A. Vladikas, Nucl. Phys. B358, 212 (1991).

[8] M. Lüscher, S. Sint, R. Sommer, and P. Weisz, Nucl. Phys. B478, 365 (1996).

[9] M. Lüscher, S. Sint, R. Sommer, P. Weisz, and U. Wolff, Nucl. Phys. B491, 323 (1997).

[10] P. H. Ginsparg and K. G. Wilson, Phys. Rev. D 25, 2649 (1982).

[11] P. Hasenfratz and F. Niedermayer, Nucl. Phys. B414, 785 (1994).

[12] W. Bietenholz and U. J. Wiese, Nucl. Phys. B464, 319 (1996).

[13] P. Hasenfratz, S. Hauswirth, K. Holland, T. Jörg, F. Niedermayer, and U. Wenger, Int. J. Mod. Phys. C 12, 691 (2001).

[14] W. Bietenholz, R. Brower, S. Chandrasekharan, and U. J. Wiese, Nucl. Phys. B, Proc. Suppl. 53, 921 (1997).

[15] T. A. DeGrand, Phys. Rev. D 58, 094503 (1998).

[16] W. Bietenholz, Eur. Phys. J. C 6, 537 (1999).

[17] C. Gattringer, Phys. Rev. D 63, 114501 (2001).

[18] C. Gattringer, I. Hip, and C. B. Lang, Nucl. Phys. B597, 451 (2001).

[19] J. H. McClellan, in Proc. of 7th Annual Princeton Conf. on Inform. Sci. and Syst. (Dept. of Electrical Engineering, Princeton University, Princeton, New Jersey, 1973), p. 247.

[20] A. Kumar, J. Comput. Phys. 201, 109 (2004).

[21] M. Patra and M. Karttunen, Numer. Methods Partial Differ. Equ. 22, 936 (2006).

[22] C. Burden and A. N. Burkitt, Europhys. Lett. 3, 545 (1987).

[23] D. H. Adams, Phys. Lett. B 699, 394 (2011).

[24] C. Hoelbling, Phys. Lett. B 696, 422 (2011).

[25] T. A. DeGrand, A. Hasenfratz, and T. G. Kovacs (MILC Collaboration), arXiv:hep-lat/9807002.

[26] C. W. Bernard and T. DeGrand, Nucl. Phys. B, Proc. Suppl. 83, 845 (2000).

[27] M. Stephenson, C. DeTar, T. A. DeGrand, and A. Hasenfratz, Phys. Rev. D 63, 034501 (2000).
[28] J. M. Zanotti et al. (CSSM Collaboration), Phys. Rev. D 65, 074507 (2002).

[29] T. DeGrand, A. Hasenfratz, and T. G. Kovacs, Phys. Rev. D 67, 054501 (2003).

[30] S. Capitani, S. Dürr, and C. Hoelbling, J. High Energy Phys. 11 (2006) 028.

[31] M. Albanese et al. (APE Collaboration), Phys. Lett. B 192, 163 (1987).

[32] C. Morningstar and M.J. Peardon, Phys. Rev. D 69, 054501 (2004).

[33] A. Hasenfratz, R. Hoffmann, and S. Schaefer, J. High Energy Phys. 05 (2007) 029.

[34] S. Durr, Comput. Phys. Commun. 180, 1338 (2009).

[35] P. J. Moran and D. B. Leinweber, Phys. Rev. D 77, 094501 (2008).

[36] P. de Forcrand and O. Jahn, Proceedings of the Third International Workshop on Numerical Analysis and Lattice QCD, Edinburgh, June-July 2003, Lecture Notes in Computational Science and Engineering Vol. 47 (Springer, New York, 2005), p. 67.

[37] S. Dürr, Z. Fodor, C. Hoelbling, and T. Kurth, J. High Energy Phys. 04 (2007) 055.

[38] S. Necco and R. Sommer, Nucl. Phys. B622, 328 (2002).

[39] L. Del Debbio, L. Giusti, M. Lüscher, R. Petronzio, and N. Tantalo, J. High Energy Phys. 02 (2006) 011.

[40] S. Dürr et al. (BMW Collaboration), Phys. Rev. D 79, 014501 (2009).

[41] P. Hernandez, K. Jansen, and M. Lüscher, Nucl. Phys. B552, 363 (1999).

[42] T. G. Kovacs, Phys. Rev. D 67, 094501 (2003).

[43] S. Dürr, C. Hoelbling, and U. Wenger, J. High Energy Phys. 09 (2005) 030.

[44] H. Neuberger, Phys. Rev. D 57, 5417 (1998).

[45] I. Hip, T. Lippert, H. Neff, K. Schilling, and W. Schroers, Phys. Rev. D 65, 014506 (2001).

[46] W. Bietenholz, Nucl. Phys. B644, 223 (2002).

[47] U. M. Heller, F. Karsch, and B. Sturm, Phys. Rev. D 60, 114502 (1999).

[48] L. H. Karsten and J. Smit, Nucl. Phys. B183, 103 (1981).

[49] F. Wilczek, Phys. Rev. Lett. 59, 2397 (1987).

[50] R. Hoffmann, A. Hasenfratz, and S. Schaefer, Proc. Sci., LAT2007 (2007) 104 [arXiv:0710.0471].

[51] Y. Shamir, B. Svetitsky, and E. Yurkovsky, Phys. Rev. D 83, 097502 (2011).

[52] S. Capitani, Phys. Rep. 382, 113 (2003).

[53] G. I. Egri et al., Comput. Phys. Commun. 177, 631 (2007).

[54] P. Hegde, F. Karsch, E. Laermann, and S. Shcheredin, Eur. Phys. J. C 55, 423 (2008). 